\title{
LncRNA CRNDE decreases the sensitivity of sorafenib in HCC cells via promoting ATG4B- mediated autophagy
}

\section{Lingxi Chen}

Army Medical University

Liangbo Sun

Army Medical University

Xufang Dai

Chongqing Normal University

Tao Li

Army Medical University

Xiaojing Yan

Army Medical University

Yueting Zhang

Army Medical University

Hanxi Xiao

Army Medical University

Xiaodong Shen

Army Medical University

Gang Huang

Army Medical University

Wei Xiang

Army Medical University

Yan Zhang

Army Medical University

Dehong Tan

Army Medical University

Shiming Yang

Army Medical University

Yongzhan Nie

Air Force Medical University

Xuequan Huang

Army Medical University

Jiqin Lian 
Army Medical University

Fengtian He ( $\square$ hefengtian66@163.com )

Third Military Medical University

Research

Keywords: Hepatocellular carcinoma, Colorectal neoplasia differentially expressed, Autophagy related 4B, Autophagy, Sorafenib

Posted Date: February 26th, 2021

DOl: https://doi.org/10.21203/rs.3.rs-241413/v1

License: (a) This work is licensed under a Creative Commons Attribution 4.0 International License. Read Full License 


\section{Abstract}

Background: Autophagy has been confirmed to be closely related to the growth and drug resistance of cancer cells, and autophagy related 4B (ATG4B) performs a crucial role in the process of autophagy. IncRNA CRNDE (colorectal neoplasia differentially expressed) promotes the growth of hepatocellular carcinoma (HCC) cells, but it is unclear whether the tumor-promoting effect of CRNDE is associated with the regulation of ATG4B and autophagy. Methods: The expression of CRNDE and ATG4B in HCC tissues and cells was detected by quantitative real-time PCR (qPCR) and Western blot. The effect of CRNDE on autophagy of HCC cells was examined by fluorescent assay, GFP-LC3 analysis and Western blot. The relationship among CRNDE, miR-543 and ATG4B was clarified using bioinformatic analysis, qPCR, Western blot and dual-luciferase reporter assay. The effect of CRNDE on sensitivity of sorafenib in HCC cells was evaluated in vitro and in vivo by CCK-8 assay, Western blot, Hoechst staining, flow cytometry, IHC staining and TUNEL assay. Results: CRNDE induced autophagy via upregulating ATG4B in HCC cells. Mechanistically, CRNDE enhanced the stability of ATG4B mRNA by suppressing miR-543, leading to the increase of ATG4B expression and autophagy in HCC cells. Moreover, sorafenib dramatically elevated CRNDE and ATG4B as well as autophagy. Knockdown of CRNDE significantly sensitized HCC cells to sorafenib in vitro and in vivo. Conclusion: The study has revealed the presence of "CRNDE/ATG4B/autophagy" pathway which alleviates the sensitivity of sorafenib in HCC cells, suggesting that this pathway may serve as a novel target to improve the killing effect of sorafenib in HCC treatment.

\section{Background}

Hepatocellular carcinoma (HCC) is one of the most common malignant tumor in human digestive system with high incidence and low survival rate [1, 2]. The main strategies for HCC treatment with proven survival benefit include surgical resection, ablation, transplantation, chemotherapy, and so on [3]. In recent years, therapeutic reagents are increasingly used to treat $\mathrm{HCC}$, but the efficacy tends to be limited due to acquired drug resistance $[4,5]$. Autophagy, a lysosome-mediated conserved process which degrades and recycles cellular proteins and organelles in response to starvation or cellular stresses, plays an essential part in maintaining intracellular homeostasis. Mounting studies have verified that autophagy attenuates the sensitivity of therapeutic drugs such as sorafenib, 5-fluorouracil (5-FU) and oxaliplatin, ultimately protecting cancer cells from death [6-8]. Therefore, it is of great significance to understand the specific regulating mechanisms of autophagy in drug resistance and interfere with protective autophagy in order to improve the drug sensitivity in HCC.

Autophagy related 4B (ATG4B), a member of the autophagin protein family, performs a critical role in regulation of autophagy in mammalian cells. In the process of autophagosome formation, C-terminal arginine residue of the cytoplasmic light chain 3 (LC3) is proteolytically cleaved to generate LC3-I, which subsequently conjugates with phosphatidylethanolamine and converts to membrane-bound LC3-II [9]. The cysteine protease ATG4 is essential for activation of LC3 precursor and delipidation of LC3-II. Among the four isoforms of ATG4 (4A, 4B, 4C and 4D), ATG4B exhibits the dominant proteolysis activity in 
autophagic homeostasis [10]. Recent studies have shown that ATG4B promotes the proliferation and progression of various tumor cells, and enhances the drug resistance of HCC cells through protective autophagy [11-14]. However, it is not well known about the molecular mechanisms underlying the regulation of ATG4B in HCC cells.

Long noncoding RNAs (IncRNAs), a subgroup of non-encoding transcripts longer than 200 nucleotides in length, perform functions of regulating gene expression directly or indirectly at several levels such as transcriptional, post transcriptional, translational and epigenetic regulation [15]. An increasing number of IncRNAs have been reported to play a vital role in the carcinogenesis and development of HCC [16, 17]. As a new member of IncRNA family, CRNDE (colorectal neoplasia differentially expressed) is initially identified as the specifically upregulated IncRNA in human colorectal cancer, and afterwards confirmed to be highly expressed in many other human malignancies including HCC [18-20]. CRNDE promotes the growth and invasion of HCC cells by regulating multiple signalling pathways, such as PI3K/Akt, BCAT1, MAPK and Wnt/ $\beta$-catenin signalling pathways [21-24], suggesting that CRNDE may act as an oncogenic IncRNA which could be served as a potential target for HCC therapy [25]. Nevertheless, whether CRNDE is associated with ATG4B and autophagy, and the roles of CRNDE in HCC cells in response to therapeutic agents remain unclear.

In the present study, we for the first time demonstrated that CRNDE induced autophagy of HCC cells via upregulating ATG4B. Mechanistic research showed that CRNDE enhanced the stability of ATG4B mRNA by suppressing miR- 543 , thus elevating ATG4B and autophagy in HCC cells. Furthermore, sorafenib promotes CRNDE and ATG4B as well as autophagy, while inhibiting the "CRNDE/ATG4B/autophagy" pathway sensitized HCC cells to sorafenib. The in vivo experiments in nude mice showed that knockdown of CRNDE dramatically strengthened the anti-HCC effect of sorafenib. Together, these findings suggest that the pathway "CRNDE/ATG4B/autophagy" may be a promising target to increase the sensitivity of sorafenib against HCC.

\section{Materials And Methods}

\section{Clinical specimens}

Human HCC specimens and the adjacent noncancerous tissues were collected from Southwest Hospital of Army Medical University (Third Military Medical University) (Chongqing, China).

\section{TCGA(The Cancer Genome Atlas) data}

HCC RNA-Seq data and the related clinical data were from TCGA database (http://cancergenome.nih.gov/).

\section{Cell culture}

Human HCC cell lines PLC, HepG2 and Hep3B were purchased from the American Type Culture Collection (Manassas, VA, USA). Huh7, SMMC7721 cell lines and hepatic cell line THLE-3 were from the Cell Bank of 
Chinese Academy of Sciences (Shanghai, China). The cells were cultured in high-glucose DMEM (Gibco, Carlsbad, CA, USA) containing $10 \% \mathrm{FBS}$ at $37{ }^{\circ} \mathrm{C}$ in a humid incubator with $5 \% \mathrm{CO}_{2}$.

\section{Quantitative real-time PCR (qPCR)}

Total RNA from HCC cells or tissues was extracted using the total RNA extraction kit (BioFlux, Hangzhou, China) according to the manufacturer's instructions. For nuclear/cytoplasmic separation assay, cytoplasmic and nuclear RNA were separately isolated using PARIS kit (Invitrogen, Carlsbad, CA, USA) according to the manufacturer's protocol. The RNA was reversely transcribed to first-strand cDNA using PrimeScript RT reagent kit (Takara, Dalian, China). qPCR was performed with SYBR qPCR master mix (Takara), taking GAPDH as the internal reference. Reverse transcription and qPCR of miRNAs were conducted with All-in-One miRNA qRT-PCR detection kit (GeneCopoeia, Guangzhou, China), taking U6 RNA as the internal reference. The primers were listed in Supplementary Table 1.

\section{Western blot}

Total proteins from HCC cells or tissues were extracted with RIPA lysis buffer (Beyotime, Shanghai, China) and the concentrations were detected using BCA protein assay kit (Beyotime). Then Western blot was performed as previously described [6]. The primary antibodies anti-ATG4B and anti-GAPDH were from Proteintech (Chicago, IL, USA), anti-PARP and anti-SQSTM1/p62 were from Cell Signaling Technology (CST, Beverly, MA, USA), and anti-LC3 was from Sigma-Aldrich (St Louis, MO, USA).

\section{Construction of plasmids}

The DNA fragments encoding the wild type and the mutant human CRNDE containing mutations of the predicted miR-543 binding site (1008 CTTTAT $\underline{\text { TGGATT }}$ AAT GAATGTTT 1031, the underlined nucleotides were mutated) were chemically synthesized by Sangon Biotech (Shanghai, China), and separately inserted into pcDNA3.1(+) (pcDNA3.1) expression vector (Invitrogen, Carlsbad, CA, USA) after digestion with ECoR囚 (Takara) and BamH囚 (Takara). The reconstructed plasmids were named as pcDNA-CRNDE and pcDNA-CRNDE-mut, respectively. The DNA fragments encoding the wild type and the mutant 3区-UTR of human ATG4B mRNA containing mutations of the putative miR-543 binding site (1565 TGTCAGACACAGACATGAATTICI 1588, the underlined nucleotides were mutated) were separately synthesized by Sangon Biotech, digested with Sac》(Takara) and Saß (Takara), and then cloned into pmirGLO reporter vector (Thermo Scientific, Waltham, MA, USA). The reconstructed plasmids were named as pmir-ATG4B and pmir-ATG4B-mut, respectively. The overexpression plasmid pCMV-ATG4B was bought from Lab Cell Biotechnology (Chongqing, China).

\section{Transfection assay}

The siRNAs targeting human CRNDE, ATG4B and control siRNA were synthesized by Lab Cell Biotechnology. Mimics and inhibitor of miRNAs, and the corresponding negative controls were purchased from GeneCopoeia. The cells were transfected using Lipofectamine 3000 (Invitrogen) according to the 
manufacturer's instructions. The sequences of siRNA were presented in Supplementary Table 2, and the sequences of miRNA mimics and inhibitor were listed in Supplementary Table 3.

\section{GFP-LC3 analysis}

The HCC cells were co-transfected with the GFP-LC3 plasmid and pcDNA-CRNDE (or pcDNA3.1) or ATG4B siRNA (or control siRNA) with Lipofectamine 3000 for $24 \mathrm{~h}$, and then fixed with $4 \%$ formaldehyde for 10 min. The cells were observed using a laser confocal immunofluorescence microscope (Carl Zeiss AG, Oberkochen, Germany). GFP-LC3 plasmid was kindly offered by Dr. N. Mizushima and Dr. T. Yoshimori (Osaka University, Japan).

\section{Fluorescent assay of autophagy}

Autophagy in live cells was analyzed using the Cyto-ID autophagy detection kit (Enzo, NY, USA) with proprietary probes specifically staining autophagosomes according to the manufacturer's protocol. Briefly, the cells were harvested, resuspended in $500 \mu \mathrm{L}$ of freshly diluted Cyto-ID green detection reagent and incubated at room temperature for $30 \mathrm{~min}$ in dark. Then fluorescence intensity was analyzed using a flow cytometer (Beckman, CA, USA).

\section{RNA FISH (fluorescence in situ hybridization)}

The Cy3-labelled probes for RNA FISH of CRNDE, 18S RNA and U6 RNA were synthesized by RiboBio (Guangzhou, China). RNA FISH was performed using the fluorescent in situ hybridization kit (RiboBio) according to the manufacturer's instructions. DAPI was used for counterstaining of nuclei. The cells were photographed under a fluorescence microscope (OlympusIX81, Tokyo, Japan).

\section{RIP (RNA immunoprecipitation) assay}

RIP assay was performed using the EZ-Magna RIP kit (Millipore/Merck, Darmstadt, German) according to the manufacturer's protocol. Briefly, cells were lysed and incubated with antibody-coated beads at $4{ }^{\circ} \mathrm{C}$ overnight. Subsequently, the co-immunoprecipitates were treated with proteinase $\mathrm{K}$ at $55^{\circ} \mathrm{C}$ for $30 \mathrm{~min}$. RNA was purified with phenol:chloroform:isoamyl alcohol (125:24:1), followed by precipitation with ethanol overnight, and reversely transcribed into CDNA using PrimeScript RT reagent kit (Takara). Then qPCR was performed with SYBR qPCR master mix (Takara).

\section{Dual-luciferase reporter assay}

HCC cells were seeded into 24-well plates and co-transfected with the indicated luciferase reporter plasmids and miRNA mimics, siRNA, expression plasmid, or the corresponding control using Lipofectamine 3000 (Invitrogen) for $24 \mathrm{~h}$. Then the cells were lysed, and the reporter assay was performed using the dual-luciferase reporter assay kit (GeneCopoeia) according to the manufacturer's instructions.

\section{Cell viability assay}


The cell viability assay was performed using cell counting kit-8 (CCK-8; Beyotime) according to the manufacture's protocol. Briefly, the cells were seeded into 48-well plates, followed by different treatments. $20 \mu \mathrm{L}$ CCK-8 reagent was added to each well and incubated at $37^{\circ} \mathrm{C}$ for $1 \mathrm{~h}$. Then the OD values at 450 $\mathrm{nm}$ were examined using a microplate reader (Molecular Devices, Sunnyvale, CA, USA).

\section{Cell apoptosis detection}

Cell apoptosis was detected using the following methods: (1) The cells were fixed with $4 \%$ paraformaldehyde for 10 min and stained with Hoechst 33258 (Beyotime) for $10 \mathrm{~min}$ in dark. Then the cells were photographed under a fluorescence microscope (OlympusIX81) and the apoptotic cells were characterized by the nuclear morphology changes. (2) The cells were harvested and stained with Annexin\/FITC and PI (BD, California, USA) at room temperature for $15 \mathrm{~min}$ in dark. The apoptotic cells were analyzed under a flow cytometer (Beckman).

\section{Animal experiments}

Male nude mice (six weeks old) were obtained from Beijing Huafukang Bioscience (Beijing, China). Lentivirus system was used to construct the HepG2 cell lines with (or without) stable knockdown of CRNDE (LV-shCRNDE or LV-NC) as previously described [6]. $5 \times 10^{6}$ cells in $0.1 \mathrm{~mL}$ PBS (for each mouse) were subcutaneously injected into the right flank of each mouse ( $n=10$ per group). One week after inoculation, the mice in each group were randomized into two subgroups ( $n=5$ per subgroup) and given daily administration of sorafenib (Selleckchem, Houston, USA, $30 \mathrm{mg} / \mathrm{kg}$ ) or vehicle control by gavage. The size of xenograft tumors was measured every three days and the volume was calculated with the following formula: volume $=$ width $^{2} \times$ length $\times 1 / 2$. Twenty-five days after inoculation, the mice were sacrificed and the tumors were excised for photographing and weighting. The cell apoptosis in the xenograft tumors was determined using TUNEL staining, and the levels of corresponding RNAs and proteins were analysed by immunohistochemical (IHC) staining, qPCR and Western blot, respectively.

\section{IHC staining and TUNEL assay}

The xenograft tumor tissues were fixed with $4 \%$ paraformaldehyde, and then embedded in paraffin and sectioned. Next, the tumor sections were immunostained using histostain-plus kit (Zhongshan Biotechnologies) according to the manufacturer's instructions, counterstained with hematoxylin and photographed under a microscope (OlympusIX81). For apoptosis analysis of the xenograft tumors, TUNEL assays were conducted according to the manufacturer's protocol (Zhongshan Biotechnologies) and the tumor sections were visualized using a laser confocal microscope (Carl Zeiss AG).

\section{Statistical analysis}

The data were presented as means \pm SD. Two-tailed unpaired $t$-test was used for the comparisons between two independent groups and one-way analysis of variance (ANOVA) was for the comparisons 
among three or more groups. Pearson's test was employed for the analysis of the correlation between CRNDE and ATG4B levels. $P<0.05$ was considered statistically significant.

\section{Results}

\section{CRNDE upregulates ATG4B in HCC cells}

To preliminarily explore the relationship between CRNDE and ATG4B, TCGA database was used to analyze the levels of CRNDE and ATG4B in HCC tissues. The results showed that the levels of CRNDE and ATG4B mRNA in HCC tissues were significantly higher than those in noncancerous liver tissues (Fig. 1a, b). The correlation analysis revealed that the level of ATG4B was positively correlated to that of CRNDE in the HCC tissues (Fig. 1c). Furthermore, both CRNDE and ATG4B mRNA levels were associated with a shorter overall survival of HCC patients (Supplementary Fig. S1a, b). The results of qPCR (Fig. 1d, e) and Western blot (Fig. 1f) showed that the levels of CRNDE as well as ATG4B mRNA and protein in HCC tissues were higher than those in the corresponding adjacent noncancerous liver tissues. In addition, the levels of CRNDE and ATG4B in 5 HCC cell lines (SMMC-7721, HepG2, Hep3B, Huh7 and PLC) were also higher than those in relatively normal hepatic cell line THLE-3 (Supplementary Fig. S2a, b). To investigate whether ATG4B could be regulated by CRNDE, CRNDE was overexpressed and silenced in HCC cells (Supplementary Fig. S3a, b). Overexpression of CRNDE markedly increased ATG4B at both mRNA and protein levels (Fig. 1g, h), whereas silence of CRNDE dramatically reduced the mRNA and protein levels of ATG4B in HCC cells (Fig. 1i, j). Taken together, these results indicate that CRNDE elevates ATG4B in HCC cells.

\section{CRNDE enhances autophagy via upregulating ATG4B}

As ATG4B has dual roles in regulating LC3 system (either promoting or suppressing the formation of autophagosome) [26], we investigated the effect of ATG4B on autophagy in HCC cells. siRNAs targeting ATG4B were used to silence ATG4B expression in HCC cells (Supplementary Fig. S4). As shown in Fig. 2a, overexpression of ATG4B increased LC3-II while decreased SQSTM1/p62, whereas knockdown of ATG4B reduced LC3-II while elevated SQSTM1/p62. Next, we assayed whether CRNDE-mediated upregulation of ATG4B could affect autophagy in HCC cells. As shown in Fig. 2b-e, overexpression of CRNDE significantly increased fluorescence intensity of autophagosome (Fig. 2b), GFP-LC3 puncta (Fig. 2c, d) and LC3-II accumulation (Fig. 2e), simultaneously decreased SQSTM1/p62 (Fig. 2e) in HCC cells. While knockdown of CRNDE obviously declined LC3-II and elevated SQSTM1/p62 (Supplementary Fig. S5a). Moreover, autophagy inhibitor chloroquine (CQ) dramatically enhanced CRNDE-induced LC3-II accumulation and reversed CRNDE-mediated SQSTM1/p62 reduction (Fig. 2f). Since LC3-II and SQSTM1/p62 are widely used as markers of autophagic flux, these results indicated that CRNDE induced autophagy flux in HCC cells. Furthermore, silence of ATG4B remarkably ameliorated the CRNDE-increased fluorescence intensity of autophagosome (Fig. 2g), GFP-LC3 puncta (Fig. 2h and i) and LC3-II accumulation (Fig. 2j), while abolished the CRNDE-mediated decline of SQSTM1/p62 (Fig. 2j). Additionally, overexpression of ATG4B alleviated the decrease of LC3-II and increase of SQSTM1/p62 caused by CRNDE knockdown 
(Supplementary Fig. S5b). In brief, the above data suggest that CRNDE promotes autophagy through upregulating ATG4B in HCC cells.

\section{CRNDE promotes ATG4B mRNA stability}

In order to inquiry the mechanism by which CRNDE elevates ATG4B mRNA and protein levels in HCC cells, RNA FISH and nuclear/cytoplasmic separation assay were used to detect the subcellular localization of CRNDE. As shown in Fig. 3a, b, the majority of CRNDE distributed in cytoplasm and the minority located in nucleus. It has been reported that IncRNAs could directly bind to the corresponding proteins to exert their functions [27], thus RIP was performed to verify whether CRNDE could directly bind to ATG4B protein. However, the result showed that CRNDE was not specifically enriched in the coimmunoprecipitated RNAs with ATG4B (Fig. 3c). Subsequently, we examined the effect of CRNDE on the mRNA stability of ATG4B in HCC cells. In the presence of the transcription inhibitor actinomycin D (Act D), the level of ATG4B mRNA in CRNDE-overexpressed cells was significantly higher than that in the control cells (Fig. 3d). Conversely, the level of ATG4B mRNA in CRNDE-knockdown cells was markedly lower than that in the corresponding control (Fig. 3e). These findings reveal that CRNDE upregulates ATG4B through increasing its mRNA stability in HCC cells.

\section{miR-543 targets and represses ATG4B}

Previous research shows that cytoplasmic IncRNA can function as a competing endogenous RNA (ceRNA) by sequestrating miRNA, thereby regulate the expression of specific mRNA targets [28, 29]. As CRNDE mainly locates in cytoplasm and influences the stability of ATG4B mRNA, we hypothesized that miRNAs might participate in the CRNDE-mediated regulation of ATG4B in HCC cells. Thus, the miRNAs potentially binding to both CRNDE and ATG4B were predicted using online bioinformatic tools StarBase (http://starbase.sysu.edu.cn/) and TargetScan (http://www.targetscan.org/vert_72/). Eight candidate miRNAs were picked out from the putative miRNAs and identified whether CRNDE was involved in their regulation. As shown in Fig. 4a, overexpression of CRNDE decreased the levels of miR-543, miR-126-5p and miR-384 in HepG2 and Hep3B cells. In contrast, knockdown of CRNDE increased the expression of these three miRNAs (Fig. 4b). Next, we determined whether these three miRNAs could affect the expression of ATG4B. As shown in Fig. 4c, only miR-543 mimics could dramatically repress ATG4B expression, while miR-543 inhibitor elevated both the mRNA and protein levels of ATG4B (Fig. 4d, e). Bioinformatic analysis showed that miR-543 might bind to CRNDE and target 3囚-UTR of ATG4B mRNA, and the potential binding sites were shown in Fig. $4 \mathrm{f}$. Furthermore, luciferase reporter assays revealed that miR-543 mimics obviously reduced the luciferase activity of pmir-ATG4B, but not pmir-ATG4B-mut (in which the binding site of miR-543 in 3囚-UTR of ATG4B mRNA was mutated) (Fig. 4g). Collectively, these results indicated that miR-543 directly targets 3囚-UTR of ATG4B mRNA and inhibits ATG4B.

\section{CRNDE elevates ATG4B and autophagy via sequestrating miR-543}

To clarify whether miR-543 exerts essential effect on CRNDE-mediated elevation of ATG4B, CRNDE overexpression plasmid and miR-543 mimics were co-transfected into HCC cells. miR-543 remarkably 
alleviated the CRNDE-triggered upregulation of ATG4B at both mRNA (Fig. 5a) and protein (Fig. 5b) levels, and attenuated CRNDE-enhanced ATG4B mRNA stability in HCC cells (Fig. 5c). Luciferase reporter assays showed that overexpression of CRNDE increased the luciferase activity of pmir-ATG4B (Fig. 5d), while silence of CRNDE decreased its luciferase activity (Fig. 5e). Additionally, miR-543 markedly impaired the CRNDE-induced luciferase activity of pmir-ATG4B (Fig. 5f). Moreover, compared to pcDNA-CRNDE, pcDNA-CRNDE-mut (in which the binding site of miR-543 was mutated) had no significant effect on miR543 level (Fig. 5g), but led to an attenuated induction of ATG4B mRNA (Fig. 5h) and protein (Fig. 5i) as well as LC3-II (Fig. 5i), while caused a weakened reduction of SQSTM1/p62 (Fig. 5i). These data indicated that CRNDE sequestrates miR-543, leading to the upregulation of ATG4B and enhancement of autophagy.

\section{The "CRNDE/ATG4B/autophagy" pathway alleviates the sensitivity of sorafenib in HCC cells}

As shown in Supplementary Fig. S6a, b, overexpression of CRNDE promoted the viability of HCC cells while silence of CRNDE suppressed the cell viability, which was consistent with the previous reports [23, $30,31]$. Since protective autophagy tends to weaken the anti-tumor effect of therapeutic reagents, we further investigated whether the CRNDE-triggered autophagy influenced the response of HCC cells to sorafenib. Interestingly, we found that sorafenib dramatically upregulated CRNDE (Fig. 6a) and ATG4B (Fig. 6b, c), increased LC3-II while decreased SQSTM1/p62 (Fig. 6c) in HCC cells. Additionally, knockdown of CRNDE or ATG4B, or inhibition of autophagy with CQ strengthened the suppression effect of sorafenib on cell survival (Fig. 6d), and increased sorafenib-induced PARP cleavage (Fig. 6e), apoptotic bodies (Fig. 6f) and apoptotic cells (Fig. 6g). Furthermore, overexpression of ATG4B markedly attenuated the reduction of cell survival caused by the co-treatment with si-CRNDE and sorafenib (Fig. 6h). The decrease of cell survival caused by si-CRNDE (or si-ATG4B) combined with sorafenib was abolished by autophagy inducer rapamycin (RAPA) (Fig. 6i), but not affected by autophagy inhibitor CQ (Fig. 6j) co-treatment. Nevertheless, inhibition of autophagy with CQ significantly alleviated CRNDE (or ATG4B)-increased cell survival in the presence of sorafenib (Fig. $6 \mathrm{k}$ ). Altogether, these results prove that sorafenib activates the "CRNDE/ATG4B/autophagy" pathway, which alleviates the sensitivity of sorafenib in HCC cells.

\section{Knockdown of CRNDE enhances the anti-HCC effect of sorafenib in vivo}

To validate whether the above phenomenon is present in vivo, the xenograft tumor models with (or without) stable knockdown of CRNDE were established in nude mice (Supplementary Fig. S7a, b). Knockdown of CRNDE remarkably strengthened the sorafenib-mediated inhibition of xenograft tumor growth (Fig. 7a-C) and decrease of the cell proliferation marker Ki67 (Fig. 7 d), while increased sorafenibtriggered cell apoptosis (Fig. 7 e) and PARP cleavage (Fig. 7g) in HCC xenograft tumors. Sorafenib significantly elevated the levels of CRNDE (Fig. 7f) and ATG4B (Fig. 7d, $f$ and g) in the xenograft tumors. Furthermore, silence of CRNDE obviously attenuated the sorafenib-induced ATG4B (Fig. 7d, $f$ and $g$ ) and LC3-II (Fig. 7g), whereas abolished the sorafenib-mediated reduction of SQSTM1/p62 (Fig. 7d and g). These results demonstrate that knockdown of CRNDE strengthens the anti-HCC effect of sorafenib in vivo. 


\section{Discussion}

In the present study, we have revealed a novel signalling pathway "CRNDE/ATG4B/autophagy", in which CRNDE upregulates ATG4B via inhibiting miR-543, leading to the enhancement of ATG4B mRNA stability and autophagy in HCC cells. Additionally, sorafenib can activate this pathway, which weakens the sensitivity of sorafenib. These findings demonstrate a promoting effect of CRNDE on regulation of autophagy and sorafenib resistance of HCC cells.

CRNDE displays a tissue-specific expression pattern, in which it has little or no expression in certain normal tissues such as liver. Several lines of evidence support the important role of CRNDE as a mediator of oncogenesis $[19,25]$. Here, we validated that CRNDE was aberrantly upregulated in HCC cell tissues and lines, which was consistent with prior studies [23,30,31]. A recent study has shown that silence of CRNDE promotes autophagy and cell viability in neurons exposed to hypoxic-ischemic [32]. However, our study indicated that overexpression of CRNDE enhanced autophagy and cell viability in HCC cells. The discrepancy probably lies in the different pathological circumstances, which deserves indepth investigation to better understand the relationship between CRNDE and autophagy.

Numerous studies have highlighted the regulation of ATG4B by enzymes, compounds, miRNAs, siRNAs, transcription factors and their binding proteins, and so on [11,33-35]. Recently, two IncRNAs have been reported to promote autophagy and chemoresistancein colorectal cancer through miR-34a/ATG4B pathway [36, 37]. Nevertheless, it is still unclear whether CRNDE is associated with the regulation of ATG4B in hepatocellular carcinoma. In this study, we for the first time verified that CRNDE at least partially accounted for ATG4B upregulation and the subsequent autophagy induction in HCC cells, which disclosed a novel signalling pathway "CRNDE/ATG4B/autophagy". Additionally, as elevated CRNDE expression is typically found in a variety of malignancies, more studies are required to explore if the CRNDE-triggered upregulation of ATG4B also exists functionally in other correlated neoplastic diseases.

RNA stability could be influenced by multiple factors such as RNA binding proteins, RNases and miRNAs [33]. Recent studies have shown that CRNDE affects the expression and/or activity of some miRNAs as a sponge $[23,24,30]$, while several miRNAs have impact on the stability of ATG4B mRNA in various cancer cells $[33,38,39]$. Here, miR-543 was screened out to be a candidate which might be involved in the CRNDE-mediated regulation of ATG4B, and subsequently proved to be necessary for "CRNDE/ATG4B/autophagy" signalling axis. Interestingly, compared to wild type CRNDE, mutation of CRNDE in the binding site of miR-543 led to an attenuated upregulation of ATG4B and LC3-II while a weakened reduction of SQSTM1/p62, suggesting that CRNDE could modulate ATG4B and the subsequent autophagy through other ways besides regulating miR-543. Additionally, the action mechanisms of miR-543 are also complicated. For instance, miR-543/TRPM7 axis participates in cervical cancer progression through PI3K/AKT and p38/MARK pathways [40]. Another study has shown that miR543/Angpt2 axis is involved in osteosarcoma metastasis and angiogenesis mediated by CTGF [41]. Hence the other miR-543-associated signalling cascades as mentioned above might be affected by the 
CRNDE-caused change of miR-543, which needs further investigation to clarify the comprehensive intracellular signal networks relevant to CRNDE and miR-543.

Sorafenib, an oral multi-kinase inhibitor, has dual anti-tumor effects on both tumor cell proliferation and angiogenesis [42]. To date, sorafenib is regarded as the first approved and most widely-used systemic drug in the treatment of advanced HCC, but the therapeutic effect is less than satisfactory largely due to acquired drug resistance $[42,43]$. Mounting evidences have shown that protective autophagy weakens the lethality of sorafenib to HCC cells $[44,45]$. Mechanistically, sorafenib can induce autophagy in HCC cells through multiple ways, such as promoting IRE1 signals [44], activating Akt pathway [46], supressing mTORC1 [47], or inhibiting STAT3/Mcl-1/Beclin 1 axis [48], and so on. However, the detailed relationship between autophagy and sorafenib has not yet been clearly clarified. Although accumulating studies have reported the significant role of CRNDE in HCC, there is currently no evidence whether CRNDE affects the sensitivity of sorafenib in HCC cells. In the present study, we elucidated that sorafenibactivated "CRNDE/ATG4B/autophagy" pathway contributed to the reduced sensitivity of sorafenib in HCC cells, which is a novel role of CRNDE to induce sorafenib resistance. Nevertheless, it remains unknown how sorafenib elevates CRNDE level. Therefore, more studies are warranted regarding the important mechanisms by which sorafenib influences CRNDE expression in HCC cells.

\section{Conclusion}

In summary, our study presents the first evidence that CRNDE upregulates ATG4B via suppressing miR543 and subsequently enhances ATG4B mRNA stability, which ultimately promotes autophagy in HCC cells. Moreover, sorafenib activates the "CRNDE/ATG4B/autophagy" pathway, and knockdown of CRNDE sensitizes HCC cells to sorafenib. These findings illustrate a key role of CRNDE in autophagy regulation and sorafenib resistance of HCC cells, and targeting the "CRNDE/ATG4B/autophagy" pathway may serve as a promising strategy to increase the sensitivity of sorafenib in HCC cells.

\section{Declarations}

\section{Ethics approval and consent to participate}

(1) Human HCC samples were obtained with informed consent. The study was approved by the Ethics Committee of Army Medical University (Third Military Medical University, Chongqing, China).

(2) All procedures in the animal experiments were performed under the guidelines of Laboratory Animal Center of Army Medical University (Third Military Medical University) and approved by the Laboratory Animal Welfare and Ethics Committee of Army Medical University (Third Military Medical University, No. 20190078).

\section{Consent for publication}

All authors have agreed to publish this manuscript. 


\section{Availability of data and material}

All data in our study are available upon request.

\section{Competing interests}

The authors declare that they have no competing interests.

\section{Funding}

This work was supported by the National Natural Science Foundation of China $(81872024,31671464$ and 82073300), the Natural Science Foundation of Chongqing (cstc2017jcyjBX0079 and cstc2018jcyjA2018), the Open Fund Research Projects of the State Key Laboratory of Cancer Biology (Air Force Medical University) (CBSKL2019ZDKF07), and the Basic and Clinical Integration Research Project of Army Medical University (Third Military Medical University) (2019JCLC04).

\section{Authors' contributions}

Lingxi Chen and Liangbo Sun performed the experiments and wrote the manuscript; Xufang Dai contributed to data curation; Tao Li and Xiaojing Yan contributed to data analysis; Yueting Zhang and Hanxi Xiao provided assist in the experiments; Xiaodong Shen and Gang Huang contributed to statistical analysis; Wei Xiang and Yan Zhang contributed to technical and material support; Dehong Tan collected clinical samples; Shiming Yang and Yongzhan Nie performed revision of the manuscript; Xuequan Huang polished the manuscript; Jiqin Lian and Fengtian He contributed to study design and funding acquisition. All authors read and approved the final paper.

\section{Acknowledgements}

We thank Dr. N. Mizushima and Dr. T. Yoshimori from Osaka University (Japan) for providing the GFP-LC3 expression vector.

\section{Abbreviations}

3囚-UTR: 3囚-untranslated region; Act D: actinomycin D; ATG4B: autophagy-related 4B cysteine peptidase; CCK-8: cell counting kit-8; CQ: chloroquine; CRNDE: colorectal neoplasia differentially expressed; FISH: fluorescence in situ hybridization; GFP: green fluorescent protein; HCC: hepatocellular carcinoma; LC3: light chain 3; IncRNA: long noncoding RNA; miRNA: microRNA; PBS: phosphate-buffered saline; qPCR: quantitative real-time polymerase chain reaction; RIP: RNA-immunoprecipitation; SQSTM1: sequestosome 1; shRNA: short hairpin ribonucleic acid; TCGA: The Cancer Genome Atlas

\section{References}


1. Bray F, Ferlay J, Soerjomataram I, Siegel RL, Torre LA, Jemal A. Global cancer statistics 2018: GLOBOCAN estimates of incidence and mortality worldwide for 36 cancers in 185 countries. CA Cancer J Clin. 2018; 68:394-424.

2. Siegel RL, Miller KD, Jemal A. Cancer statistics, 2020. CA Cancer J Clin. 2020; 70:7-30.

3. Forner A, Reig M, Bruix J. Hepatocellular carcinoma. Lancet. 2018; 391:1301-14.

4. Llovet JM, Montal R, Sia D, Finn RS. Molecular therapies and precision medicine for hepatocellular carcinoma. Nat Rev Clin Oncol. 2018; 15:599-616.

5. Yang S, Yang L, Li XY, Li BW, Li Y, Zhang XD, et al. New insights into autophagy in hepatocellular carcinoma: mechanisms and therapeutic strategies. Am J Cancer Res. 2019; 9:1329-53.

6. Xiong $\mathrm{H}, \mathrm{Ni} Z$, He J, Jiang S, Li X, He J, et al. LncRNA HULC triggers autophagy via stabilizing Sirt1 and attenuates the chemosensitivity of HCC cells. Oncogene. 2017; 36:3528-40.

7. Guo XL, Hu F, Zhang SS, Zhao QD, Zong C, Ye F, et al. Inhibition of p53 increases chemosensitivity to 5-FU in nutrient-deprived hepatocarcinoma cells by suppressing autophagy. Cancer Lett. 2014; 346:278-84.

8. Li WD, Dong XS, He CJ, Tan G, Li ZY, Zhai B, et al. LncRNA SNHG1 contributes to sorafenib resistance by activating the Akt pathway and is positively regulated by miR-21 in hepatocellular carcinoma cells. J Exp Clin Cancer Res. 2019; 38:183.

9. Mizushima N. Autophagy: process and function. Genes Dev. 2007; 21:2861-73.

10. Mizushima N. The ATG conjugation systems in autophagy. Curr Opin Cell Biol. 2019; 63:1-10.

11. Zhang N, Wu YR, Lyu XL, Li B, Yan XJ, Xiong HJ, et al. HSF1 upregulates ATG4B expression and enhances epirubicin-induced protective autophagy in hepatocellular carcinoma cells. Cancer Lett. 2017; 409:81-90.

12. Agrotis A, Ketteler R. On ATG4B as Drug Target for Treatment of Solid Tumours-The Knowns and the Unknowns. Cells. 2019; 9 (1):53.

13. Rothe K, Lin H, Lin KB, Leung A, Wang HM, Malekesmaeili M, et al. The core autophagy protein ATG4B is a potential biomarker and therapeutic target in CML stem/progenitor cells. Blood. 2014; 123:3622-34.

14. Liu PF, Chen HC, Cheng JS, Tsai WL, Lee HP, Wang SC, et al. Association of ATG4B and Phosphorylated ATG4B Proteins with Tumorigenesis and Prognosis in Oral Squamous Cell Carcinoma. Cancers (Basel). 2019; 11 (12):1854.

15. Ulitsky I, Bartel DP. lincRNAs: genomics, evolution, and mechanisms. Cell. 2013; 154:26-46.

16. Batista PJ, Chang HY. Long noncoding RNAs: cellular address codes in development and disease. Cell. 2013; 152:1298-307.

17. Huang Z, Zhou JK, Peng Y, He WF, Huang $\mathrm{CH}$. The role of long noncoding RNAs in hepatocellular carcinoma. Mol Cancer. 2020; 19:77.

18. Ellis BC, Molloy PL, Graham LD. CRNDE: A Long Non-Coding RNA Involved in CanceR, Neurobiology, and DEvelopment. Front Genet. 2012; 3:270. 
19. Xie HB, Ma B, Gao QJ, Zhan HJ, Liu YC, Chen ZC, et al. Long non-coding RNA CRNDE in cancer prognosis: Review and meta-analysis. Clin Chim Acta. 2018; 485:262-71.

20. Wang YL, Wang YT, Li JF, Zhang YZ, Yin HL,Han B, et al. CRNDE, a long-noncoding RNA, promotes glioma cell growth and invasion through mTOR signaling. Cancer Lett. 2015; 367:122-8.

21. Tang QL, Zheng XL, Zhang JK. Long non-coding RNA CRNDE promotes heptaocellular carcinoma cell proliferation by regulating PI3K/Akt /beta-catenin signaling. Biomed Pharmacother. 2018; 103:118793.

22. Zhu LY, Yang NH, Du GQ, Li CC, Liu GQ, Liu SJ, et al. LncRNA CRNDE promotes the epithelialmesenchymal transition of hepatocellular carcinoma cells via enhancing the Wnt/beta-catenin signaling pathway. J Cell Biochem. 2018; 120(2):1156-64.

23. Wang HH, Ke J, Guo QN, Nampoukime KB, Yang PW, Ma K. Long non-coding RNA CRNDE promotes the proliferation, migration and invasion of hepatocellular carcinoma cells through miR-217/MAPK1 axis. J Cell Mol Med. 2018; 22:5862-76.

24. Ji DG, Jiang CW, Zhang LR, Liang N, Jiang TC, Yang B, et al. LncRNA CRNDE promotes hepatocellular carcinoma cell proliferation, invasion, and migration through regulating miR203/BCAT1 axis. J Cell Physiol. 2019; 234:6548-60.

25. Zhang JM, Yin MN, Peng G, Zhao YC, et al. CRNDE: An important oncogenic long non-coding RNA in human cancers. Cell Prolif. 2018; 51:e12440.

26. Satoo K, Noda NN, Kumeta H, Fujioka Y, Mizushima N, Ohsumi Y, et al. The structure of Atg4B-LC3 complex reveals the mechanism of LC3 processing and delipidation during autophagy. EMBO J. 2009; 28(9):1341-50.

27. Tsagakis I, Douka K, Birds I, Aspden JL, et al. Long non-coding RNAs in development and disease: conservation to mechanisms. J Pathol. 2020; 250:480-95.

28. Cesana M, Cacchiarelli D, Legnini I, Santini T, Sthandier O, Chinappi M, et al. A long noncoding RNA controls muscle differentiation by functioning as a competing endogenous RNA. Cell. 2011; 147:35869.

29. Wang Y, Xu ZY, Jiang JF, Xu C, Kang JH, Xiao L, et al. Endogenous miRNA sponge lincRNA-RoR regulates Oct4, Nanog, and Sox2 in human embryonic stem cell self-renewal. Dev Cell. 2013; 25:6980.

30. Chen ZL, Yu C, Zhan L, Pan YZ, Chen L, Sun CY, et al. LncRNA CRNDE promotes hepatic carcinoma cell proliferation, migration and invasion by suppressing miR-384. Am J Cancer Res. 2016; 6:2299309.

31. Li ZX, Wu G, Li J, Wang YY, Ju XM, Jiang WJ. IncRNA CRNDE promotes the proliferation and metastasis by acting as sponge miR-539-5p to regulate POU2F1 expression in HCC. BMC Cancer. 2020; 20:282.

32. Fu CH, Lai FF, Chen S, Yan CX, Zhang BH, Fang CZ, et al. Silencing of long non-coding RNA CRNDE promotes autophagy and alleviates neonatal hypoxic-ischemic brain damage in rats. 2020; 472(1$2): 1-8$. 
33. Wu YR, Ni ZH, Yan XJ, Dai XF, Hu CJ, Zheng YR, et al. Targeting the MIR34C-5p-ATG4B-autophagy axis enhances the sensitivity of cervical cancer cells to pirarubicin. Autophagy. 2016; 12:1105-17.

34. Kjos I, Borg Distefano M, Saetre F, Repnik U, Holland P, Jones AT, et al. Rab7b modulates autophagic flux by interacting with Atg4B. EMBO Rep. 2017; 18:1727-39.

35. Fu YY, Hong L, Xu JC, Zhong GP, Gu Q, Gu QQ, et al. Discovery of a small molecule targeting autophagy via ATG4B inhibition and cell death of colorectal cancer cells in vitro and in vivo. Autophagy. 2019; 15:295-311.

36. Liu F, Ai FY, Zhang DC, Tian L, Yang ZY, Liu SJ. LncRNA NEAT1 knockdown attenuates autophagy to elevate 5-FU sensitivity in colorectal cancer via targeting miR-34a. Cancer Med. 2020; 9:1079-91.

37. Li YC, Li CF, Li DD, Yang L, Jin JP, Zhang B. IncRNA KCNQ10T1 enhances the chemoresistance of oxaliplatin in colon cancer by targeting the miR-34a/ATG4B pathway. Onco Targets Ther. 2019; 12:2649-60.

38. Liao HQ, Xiao Y, Hu YB, Xiao YM, Yin ZF, Liu L, et al. Methylation-induced silencing of miR-34a enhances chemoresistance by directly upregulating ATG4B-induced autophagy through AMPK/mTOR pathway in prostate cancer. Oncol Rep. 2016; 35:64-72.

39. Liu Y, Gu SJ, Li HF, Wang J, Wei CB, Liu QB. SNHG16 promotes osteosarcoma progression and enhances cisplatin resistance by sponging miR-16 to upregulate ATG4B expression. Biochem Biophys Res Commun. 2019; 518:127-33.

40. Liu XY, Gan L, Zhang J. miR-543 inhibites cervical cancer growth and metastasis by targeting TRPM7. Chem Biol Interact. 2019; 302:83-92.

41. Wang LH, Tsai HC, Cheng YC, Lin CY, Huang YL, Tsai CH, et al. CTGF promotes osteosarcoma angiogenesis by regulating miR-543/angiopoietin 2 signaling. Cancer Lett. 2017; 391:28-37.

42. Llovet JM, Ricci S, Mazzaferro V, Hilgard P, Gane E, Blanc JF, et al. Sorafenib in advanced hepatocellular carcinoma. N Engl J Med. 2008; 359:378-90.

43. Sun T, Liu HC, Ming L. Multiple Roles of Autophagy in the Sorafenib Resistance of Hepatocellular Carcinoma. Cell Physiol Biochem. 2017; 44:716-27.

44. Shi YH, Ding ZB, Zhou J, Hui B, Shi GM, Ke AW, et al. Targeting autophagy enhances sorafenib lethality for hepatocellular carcinoma via ER stress-related apoptosis. Autophagy. 2011; 7:1159-72.

45. Yi HQ, Long Bi, Ye XM, Li LF. The role and mechanism of autophagy in sorafenib targeted cancer therapy. Crit Rev Oncol Hematol. 2016; 100:137-40.

46. Zhai CG, Cheng J, Mujahid H, Wang HF, Kong J, Yin Y, et al. Selective inhibition of PI3K/Akt/mTOR signaling pathway regulates autophagy of macrophage and vulnerability of atherosclerotic plaque. PLoS One. 2014; 9:e90563.

47. Shimizu S, Takehara T, Hikita H, Kodama T, Tsunematsu H, Miyagi T, et al. Inhibition of autophagy potentiates the antitumor effect of the multikinase inhibitor sorafenib in hepatocellular carcinoma. Int J Cancer. 2012; 131:548-57. 
48. Tai WT, Shiau CW, Chen HL, Liu CY, Lin CS, Cheng AL, et al. Mcl-1-dependent activation of Beclin 1 mediates autophagic cell death induced by sorafenib and SC-59 in hepatocellular carcinoma cells. Cell Death Dis. 2013; 4:e485.

\section{Figures}

a

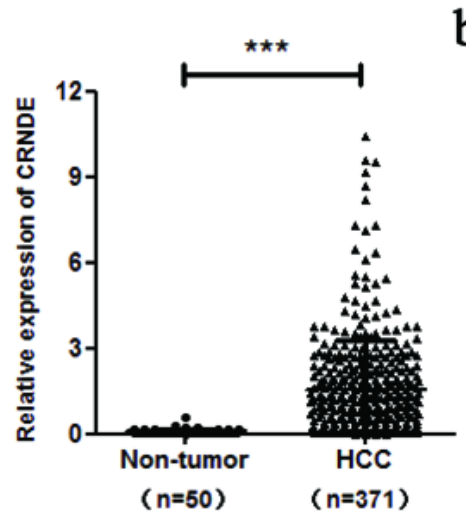

b

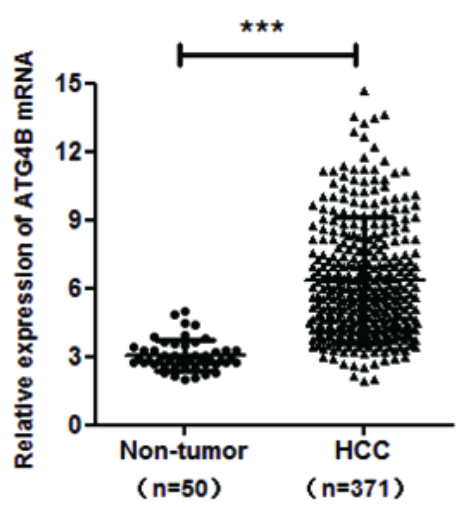

c

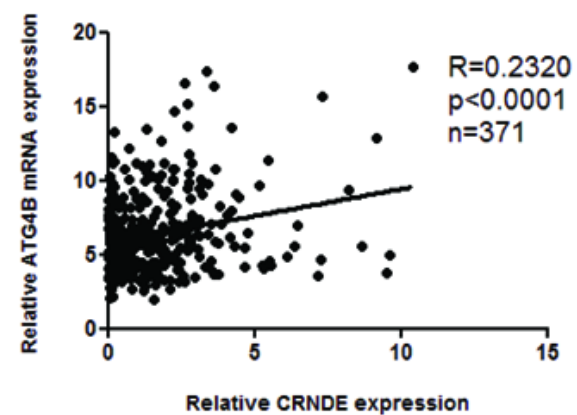

d

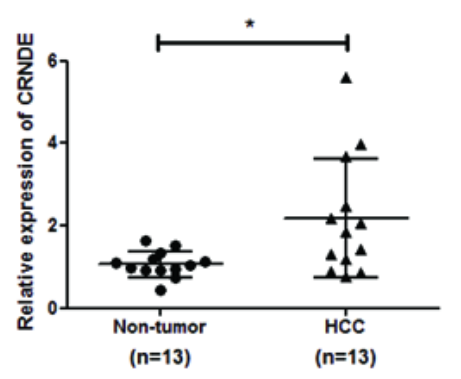

g

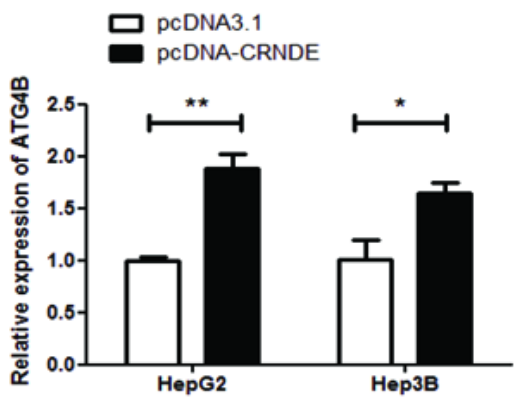

i

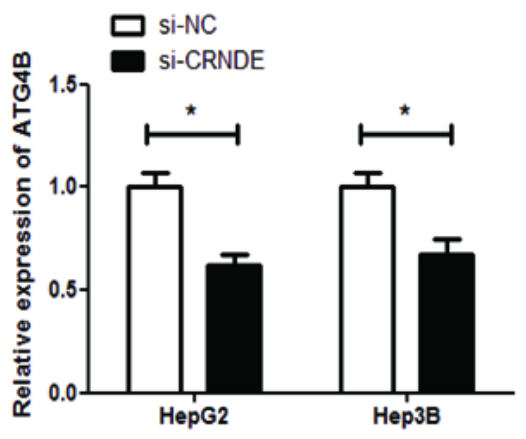

e

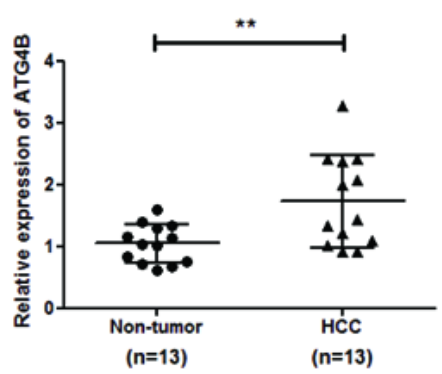

h f

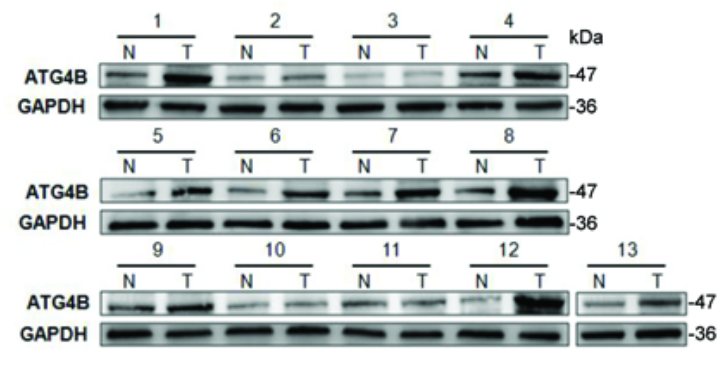

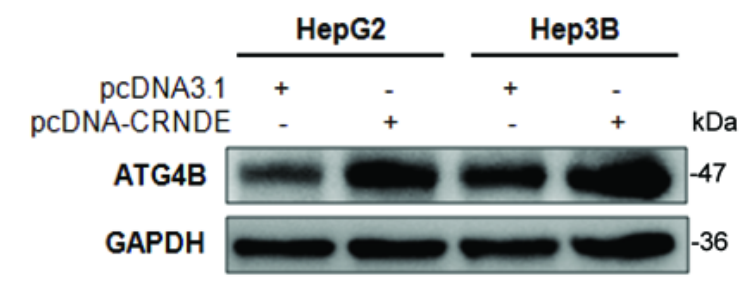

j
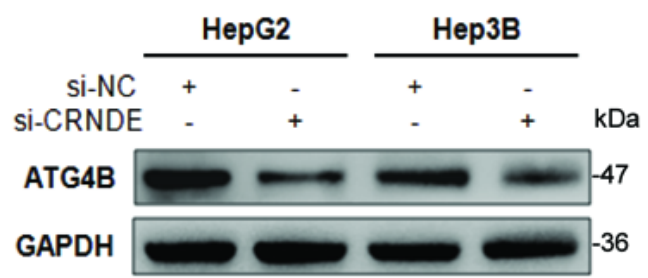

Figure 1 
CRNDE elevates ATG4B in HCC cells. a, b TCGA database was used to analyze the expression of CRNDE and ATG4B in HCC tissues $(n=371)$ and normal tissues $(n=50)$. c Correlation between CRNDE and ATG4B mRNA levels in HCC tissues $(R=0.2320, P<0.0001)$. $d$ - $f$ The levels of CRNDE and ATG4B mRNA were analyzed by qPCR, and the level of ATG4B protein was assessed by Western blot in 13 pairs of HCC tissues $(T)$ and the adjacent noncancerous liver tissues $(N)$. g, h HepG2 and Hep3B cells were transfected with pcDNA-CRNDE or pcDNA3.1. $24 \mathrm{~h}$ after transfection, the levels of ATG4B mRNA and protein were determined by qPCR and Western blot respectively. i, j $24 \mathrm{~h}$ after transfecting HepG2 and Hep3B cells with si-CRNDE or si-NC, the levels of ATG4B mRNA and protein were separately detected by qPCR and Western blot. ${ }^{*} \mathrm{P}<0.05 ;{ }^{* \star} \mathrm{P}<0.01 ;{ }^{* \star *} \mathrm{P}<0.001$. 
a

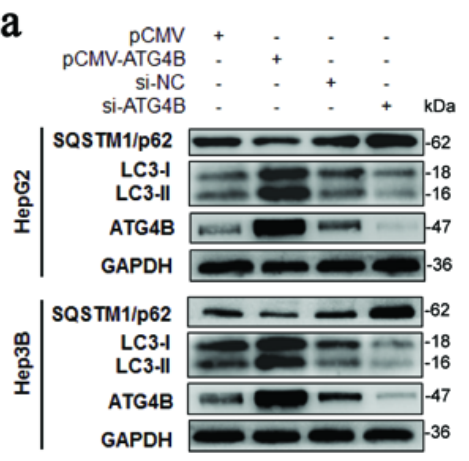

d

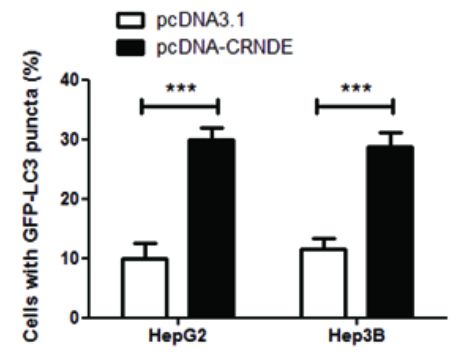

b

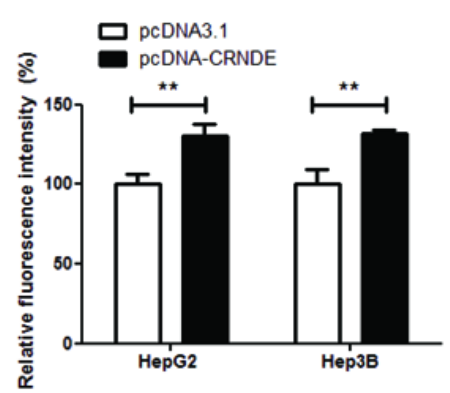

C

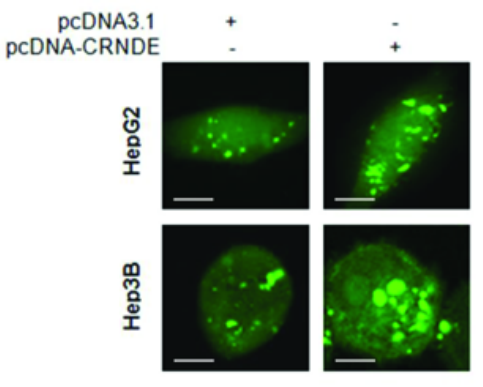

e

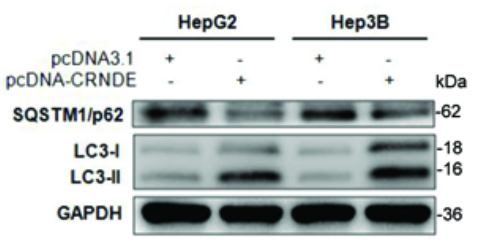

f

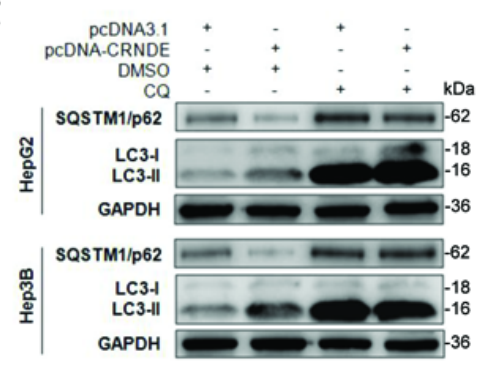

g

h
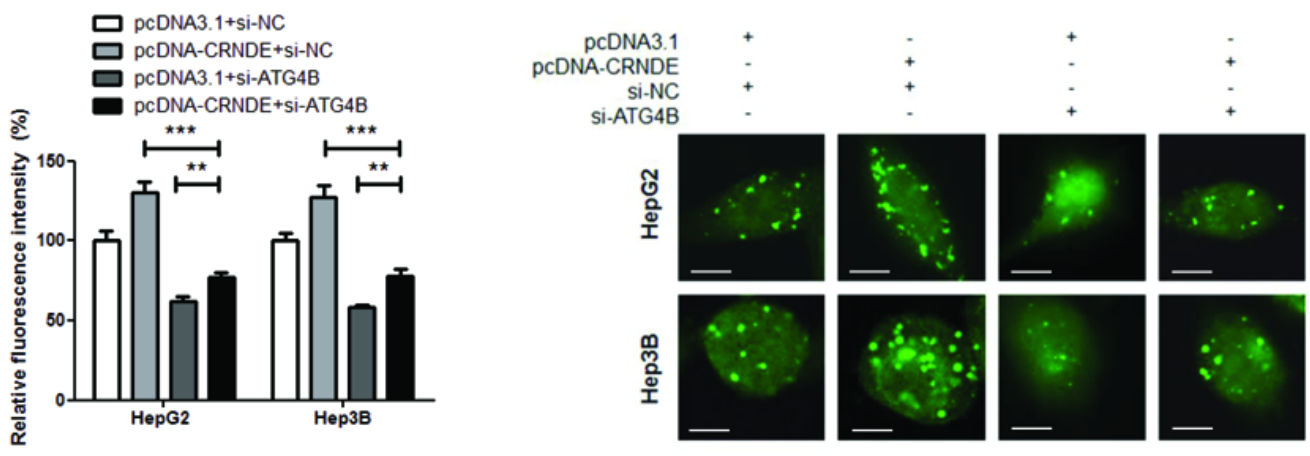

i

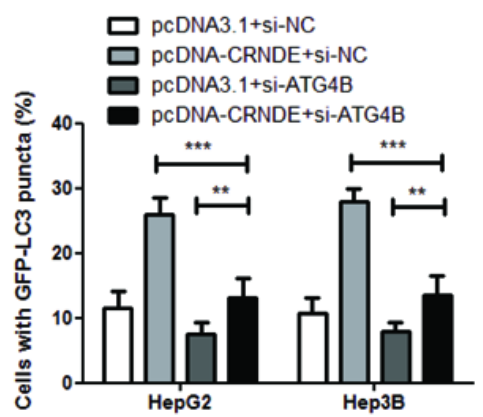

j

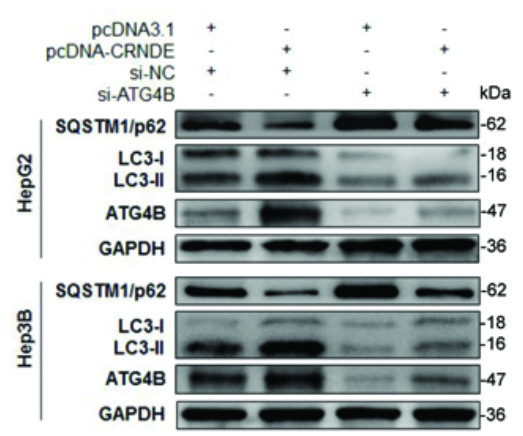

Figure 2

CRNDE induces autophagy in HCC cells through upregulating ATG4B. a HepG2 and Hep3B cells were transfected with pCMV-ATG4B, pCMV, si-ATG4B or si-NC. $24 \mathrm{~h}$ after transfection, the levels of SQSTM1/p62, LC3 and ATG4B were measured by Western blot. b $24 \mathrm{~h}$ after transfection with pcDNACRNDE or pcDNA3.1, HepG2 and Hep3B cells were stained with fluorescence probes. Subsequently, the fluorescence intensity of autophagosomes was detected using a flow cytometer. c, d HepG2 and Hep3B 
cells were co-transfected with pcDNA-CRNDE (or pcDNA3.1) and GFP-LC3 vector. $24 \mathrm{~h}$ after cotransfection, the GFP-LC3 puncta in the cells were observed under a fluorescence microscope (Scale bar: $5 \mu \mathrm{m}$ ) and the percentage of the cells containing five or more GFP-LC3 puncta was quantified. e $24 \mathrm{~h}$ after transfecting HepG2 and Hep3B cells with pcDNA-CRNDE or pcDNA3.1, the levels of SQSTM1/p62 and LC3 were examined by Western blot. $f$ HepG2 and Hep3B cells were transfected with pcDNA-CRNDE or pcDNA3.1 in the presence of $20 \mu \mathrm{M}$ CQ or vehicle control (0.1\% DMSO). $24 \mathrm{~h}$ after transfection, the levels of SQSTM1/p62 and LC3 were determined by Western blot. g $24 \mathrm{~h}$ after co-transfection with si-ATG4B (or si-NC) and pcDNA-CRNDE (or pcDNA3.1), HepG2 and Hep3B cells were stained with fluorescence probes. Then the fluorescence intensity of autophagosomes was checked using a flow cytometer. h, i HepG2 and Hep3B cells were co-transfected with si-ATG4B (or si-NC) and pcDNA-CRNDE (or pcDNA3.1) in the presence of GFP-LC3 vector. $24 \mathrm{~h}$ after co-transfection, the GFP-LC3 puncta was photographed and the percentage of the cells containing GFP-LC3 puncta was quantified as described in c and d. j $24 \mathrm{~h}$ after cotransfecting HepG2 and Hep3B cells with si-ATG4B (or si-NC) and pcDNA-CRNDE (or pcDNA3.1), the levels of SQSTM1/p62, LC3 and ATG4B were assayed by Western blot. CQ: chloroquine; ${ }^{\star *} P<0.01$; $\star \star \star P<0.001$. 
a
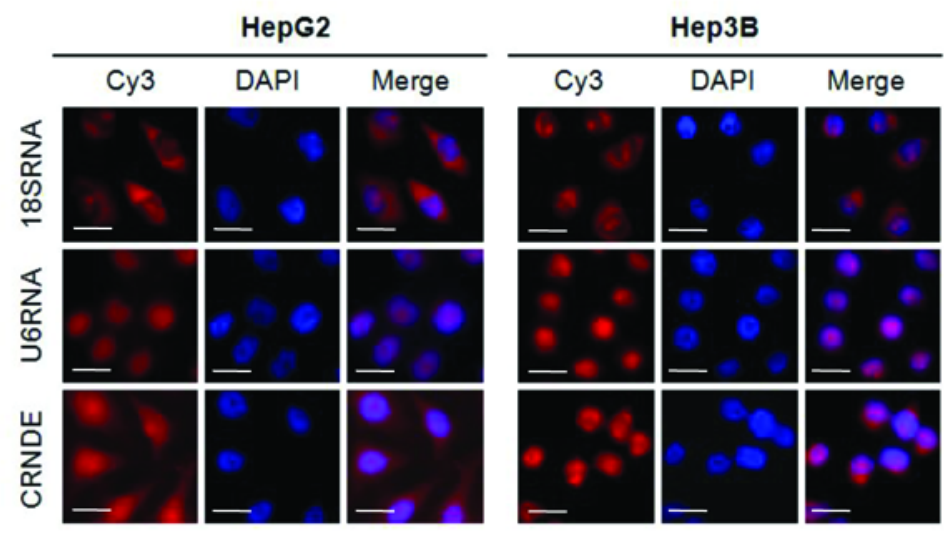

b

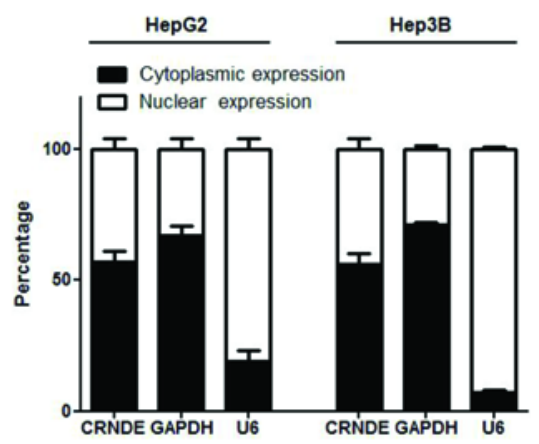

C

d

HepG2
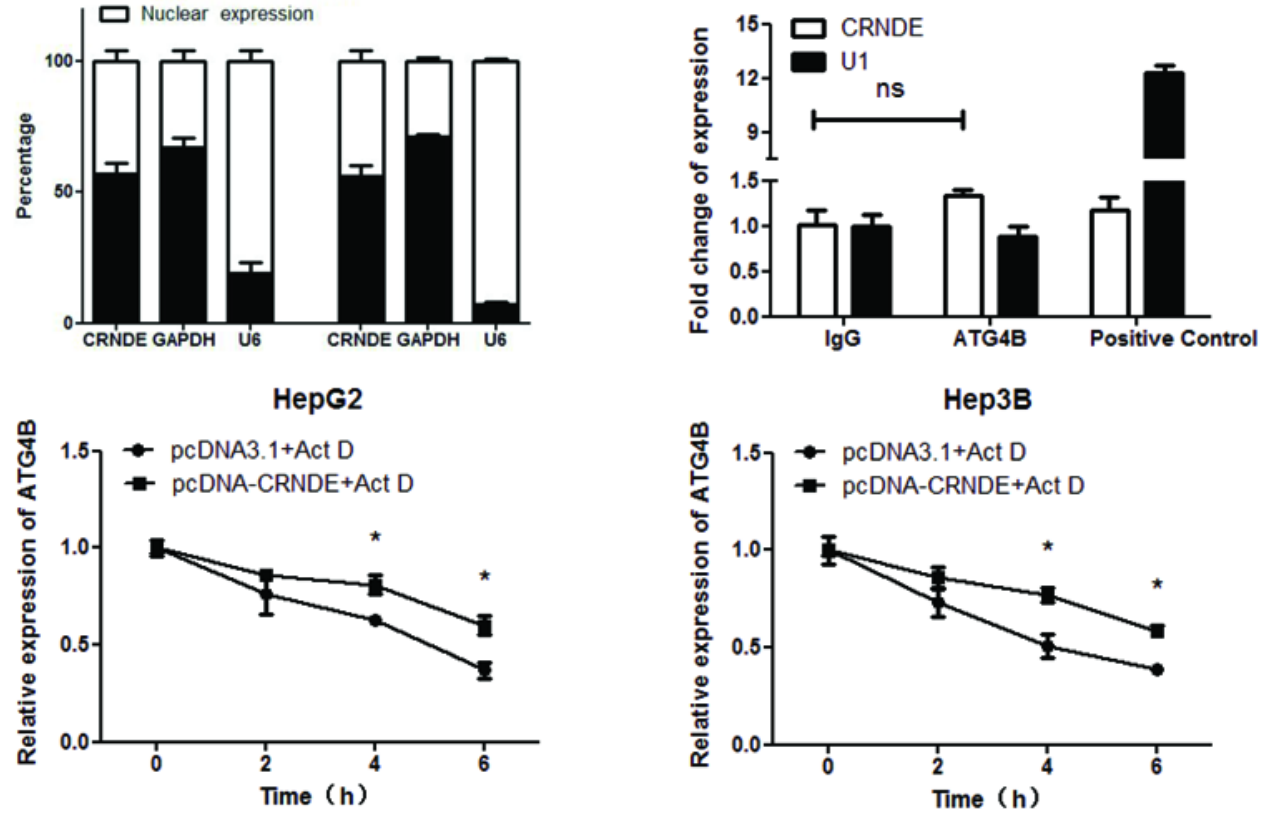

e

HepG2
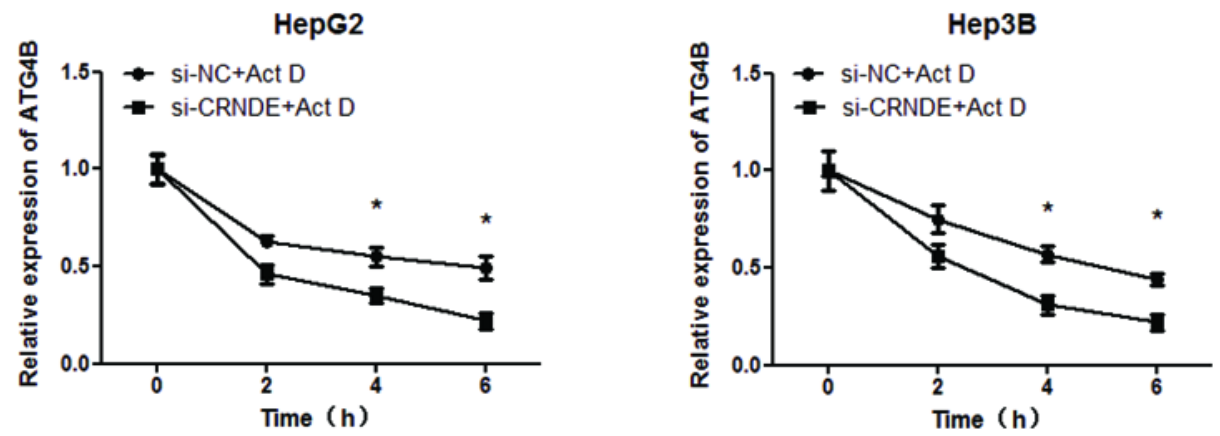

Figure 3

3CRNDE enhances the stability of ATG4B mRNA. a The subcellular distribution of CRNDE in HepG2 and Hep3B cells was tested by FISH assay. 18S RNA and U6 RNA were used as cytoplasmic and nuclear controls, respectively. The probes for CRNDE and the control RNA were labelled with Cy3 (red fluorescent signal). The nuclei were counterstained with DAPI (blue fluorescent signal). The cells were photographed under a fluorescence microscope (Scale bar: $10 \mu \mathrm{m})$. b Nuclear and cytoplasmic distribution of CRNDE in 
HepG2 and Hep3B cells was quantified by qPCR. GAPDH RNA and U6 RNA were used as cytoplasmic and nuclear controls, respectively. c RIP assay in HepG2 cells was performed with anti-ATG4B antibody. The levels of CRNDE and U1 snRNA (positive control binding RNA) in the co-immunoprecipitates were examined by qPCR. SNRNP70 and IgG were used as positive control and negative control, respectively. $d$, e $18 \mathrm{~h}$ after transfection with pcDNA-CRNDE (or pcDNA3.1) or si-CRNDE (or si-NC), HepG2 and Hep3B cells were treated with $5 \mu \mathrm{g} / \mathrm{mL}$ actinomycin $D(A c t ~ D)$ for $0,2,4$ and $6 \mathrm{~h}$. Then the level of ATG4B mRNA was examined by qPCR. ns: no significance; ${ }^{*}<<0.05$.

a
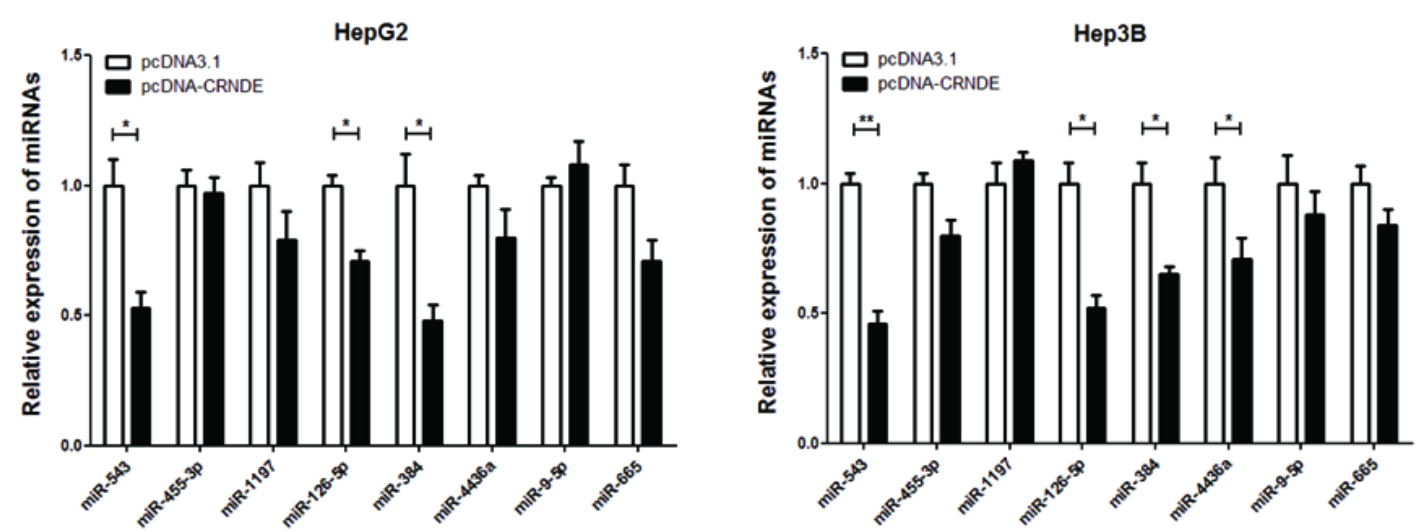

b
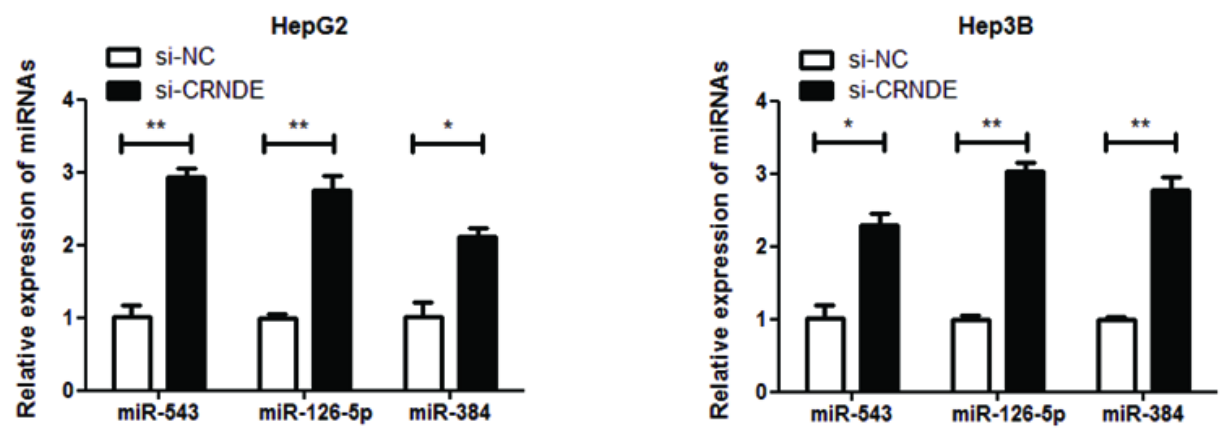

c

d

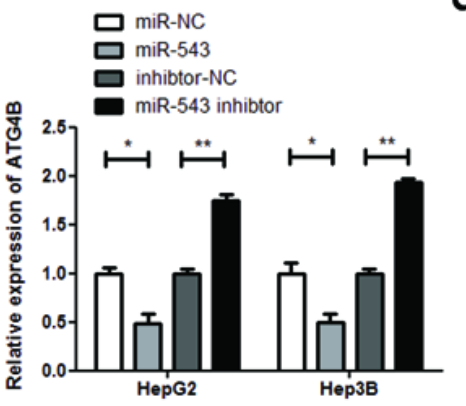

e
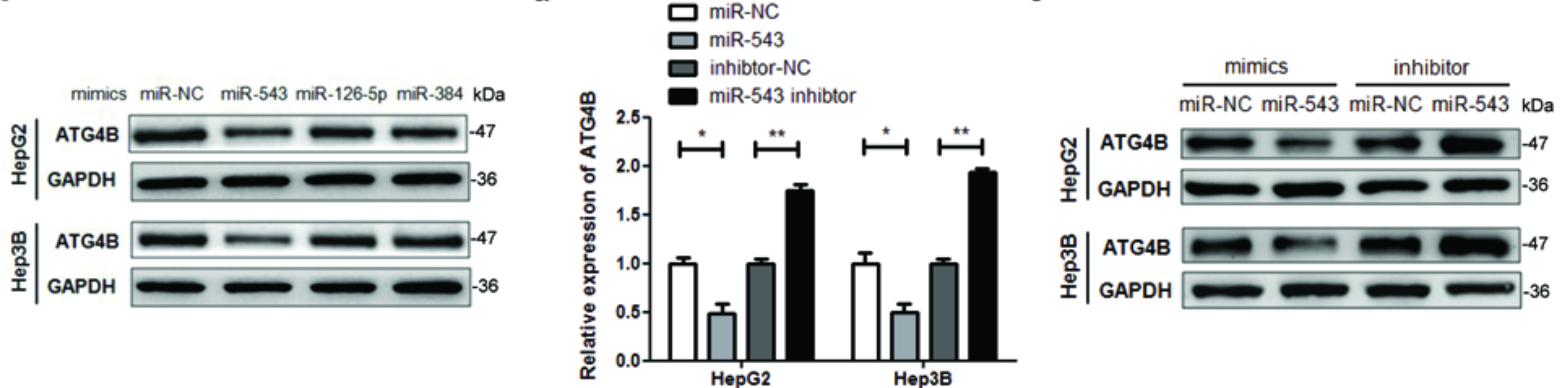

f

g
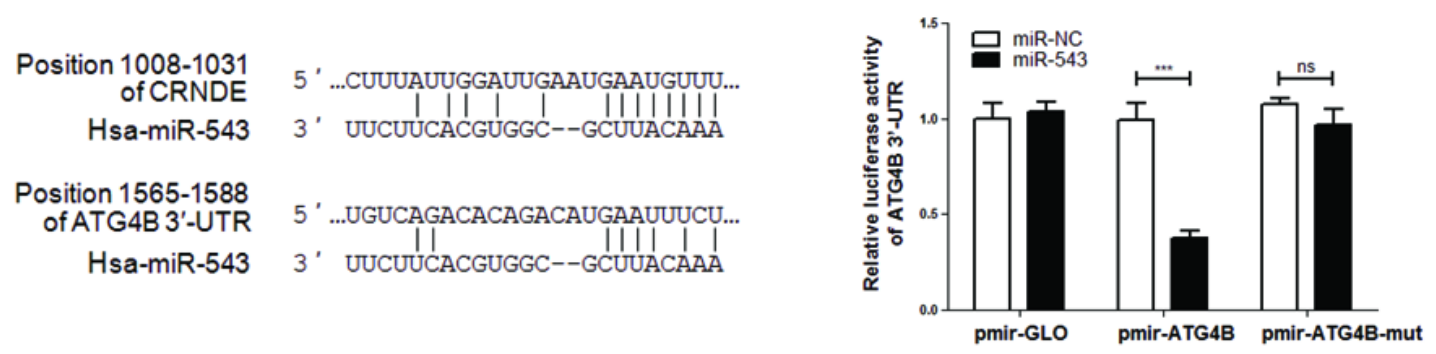


\section{Figure 4}

4miR-543 targets and inhibits ATG4B expression. a HepG2 and Hep3B cells were transfected with pcDNACRNDE or pcDNA3.1. $24 \mathrm{~h}$ after transfection, the expressions of the indicated miRNAs were detected by qPCR. b $24 \mathrm{~h}$ after transfecting HepG2 and Hep3B cells with si-CRNDE or si-NC, the levels of miR-543, miR-126-5p and miR-384 were examined by qPCR. c HepG2 and Hep3B cells were transfected with mimics of the three candidate miRNAs separately. $24 \mathrm{~h}$ after transfection, the level of ATG4B protein was measured by Western blot. d, e $24 \mathrm{~h}$ after transfecting HepG2 and Hep3B cells with miR-543 mimics, miRNC, miR-543 inhibitor or inhibitor-NC, levels of ATG4B mRNA and protein were measured by qPCR and Western blot, respectively. $f$ Prediction of the potential miR-543 binding site in CRNDE and the putative miR-543 target site in 3区-UTR of ATG4B mRNA using StarBase and TargetScan, respectively. g Reporter plasmids (pmir-ATG4B, pmir-ATG4B-mut or pmir-GLO) were transfected into HepG2 cells in the presence of miR-543 mimics or miR-NC. $24 \mathrm{~h}$ after transfection, dual-luciferase reporter assay was performed. The activity of firefly luciferase was normalized against that of rennilla luciferase. ns: no significance; ${ }^{*} \mathrm{P}<0.05 ;{ }^{* \star P}<0.01 ; * \star * P<0.001$. 
a

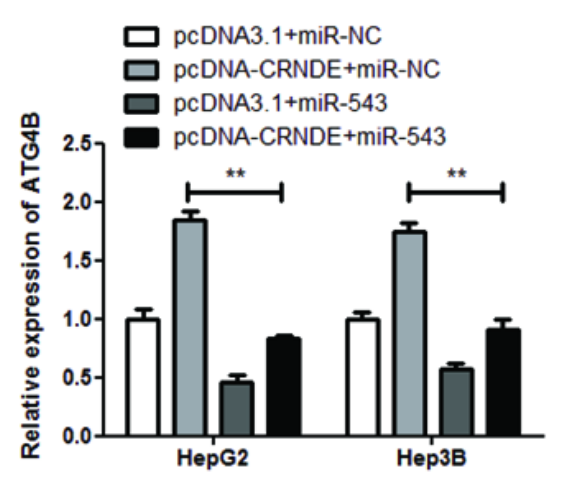

C

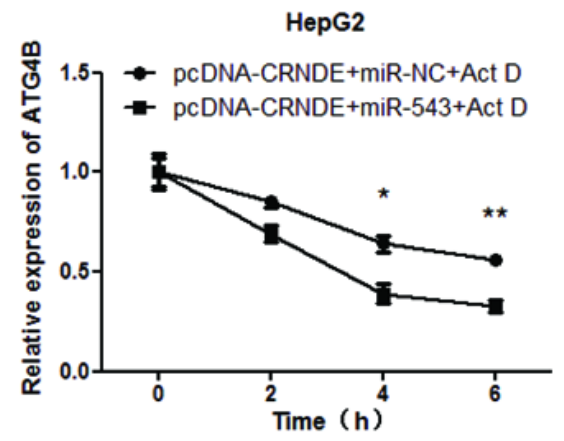

b
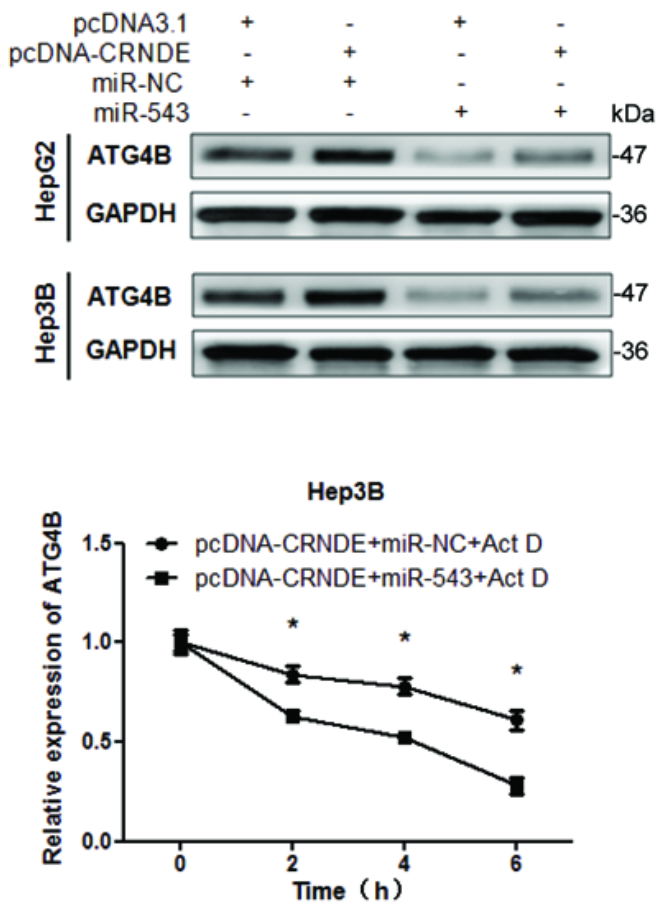

d

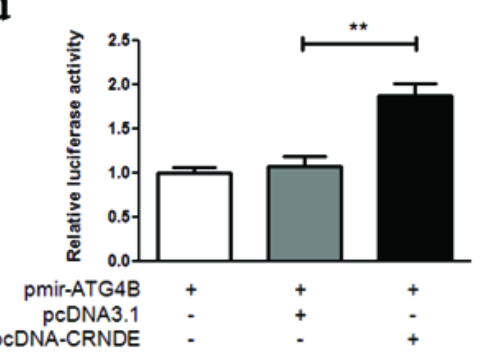

g

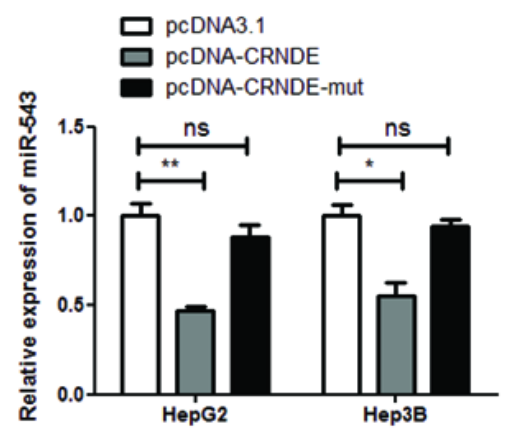

e

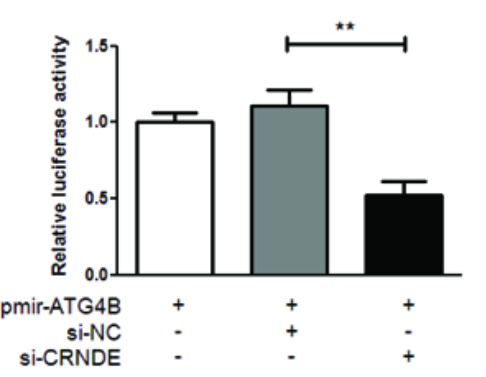

h

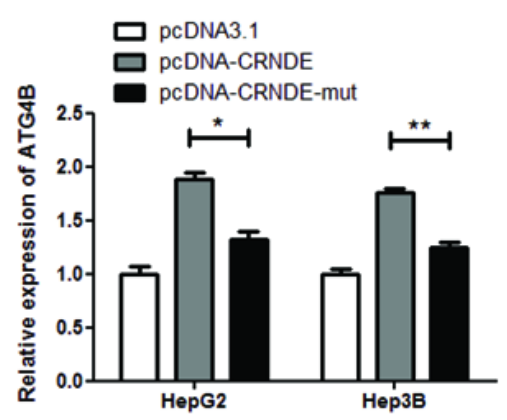

f

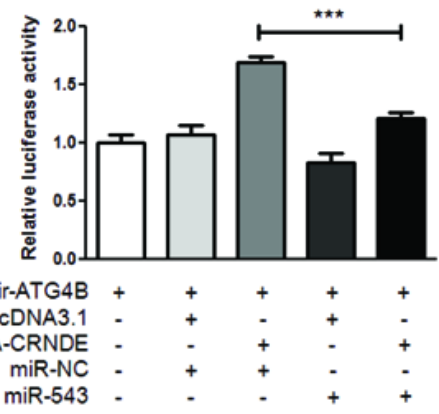

i
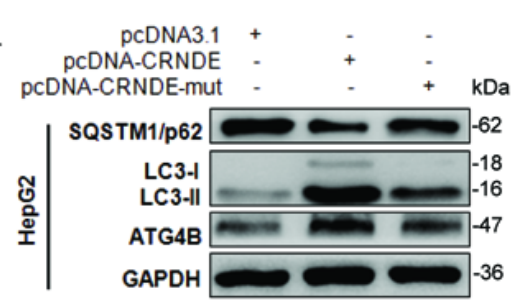

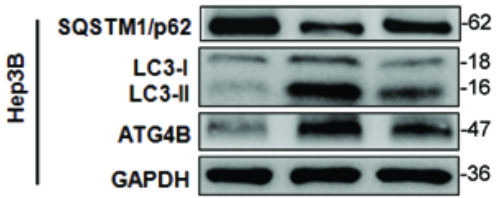

\section{Figure 5}

CRNDE promotes ATG4B expression and autophagy through sequestrating miR-543. $a, b$ HepG2 and Hep3B cells were co-transfected with pcDNA-CRNDE (or pcDNA3.1) and miR-543 mimics (or miR-NC). 24 $\mathrm{h}$ after co-transfection, mRNA and protein levels of ATG4B were determined by qPCR and Western blot, respectively. c $18 \mathrm{~h}$ after transfection with pcDNA-CRNDE and miR-543 mimics (or miR-NC), HepG2 and Hep3B cells were treated with $5 \mu \mathrm{g} / \mathrm{mL}$ actinomycin D (Act D) for 0, 2, 4 and $6 \mathrm{~h}$. Then the mRNA level of 
ATG4B was quantified using qPCR. $d$ HepG2 cells were co-transfected with pcDNA-CRNDE (or pcDNA3.1) and pmir-ATG4B. $24 \mathrm{~h}$ after transfection, dual-luciferase reporter assay was performed. The activity of firefly luciferase was normalized against that of rennilla luciferase. e HepG2 cells were co-transfected with si-CRNDE (or si-NC) and pmir-ATG4B, and the luciferase activity was assayed as described in $\mathrm{d}$. $\mathrm{f}$ HepG2 cells were co-transfected with pcDNA-CRNDE (or pcDNA3.1) and miR-543 mimics (or miR-NC) in the presence of pmir-ATG4B, and the luciferase activity was detected as in d. g-i HepG2 and Hep3B cells were separately transfected with pcDNA-CRNDE, pcDNA-CRNDE-mut, or pcDNA3.1. $24 \mathrm{~h}$ after transfection, the levels of miR-543 and ATG4B mRNA were analyzed by $q P C R$, and the level of ATG4B protein was examined by Western blot. ns: no significance; ${ }^{*} P<0.05$; ${ }^{\star *} P<0.01 ;{ }^{* \star \star} P<0.001$. 
a

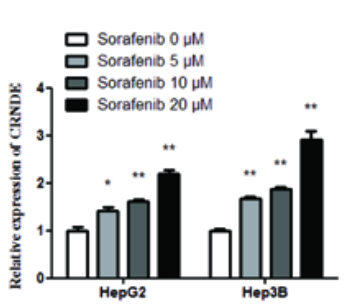

e

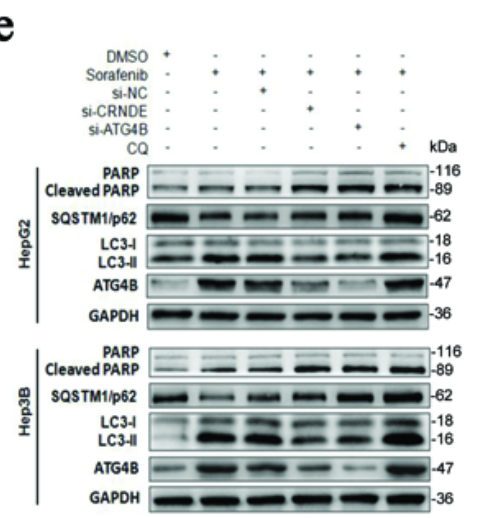

b

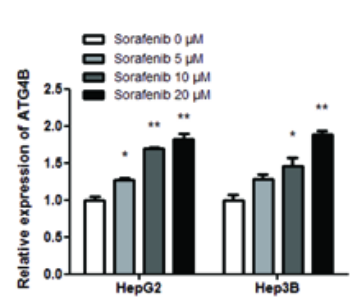

f c

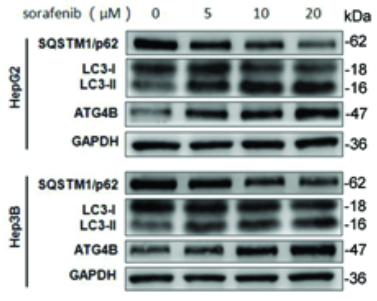

d
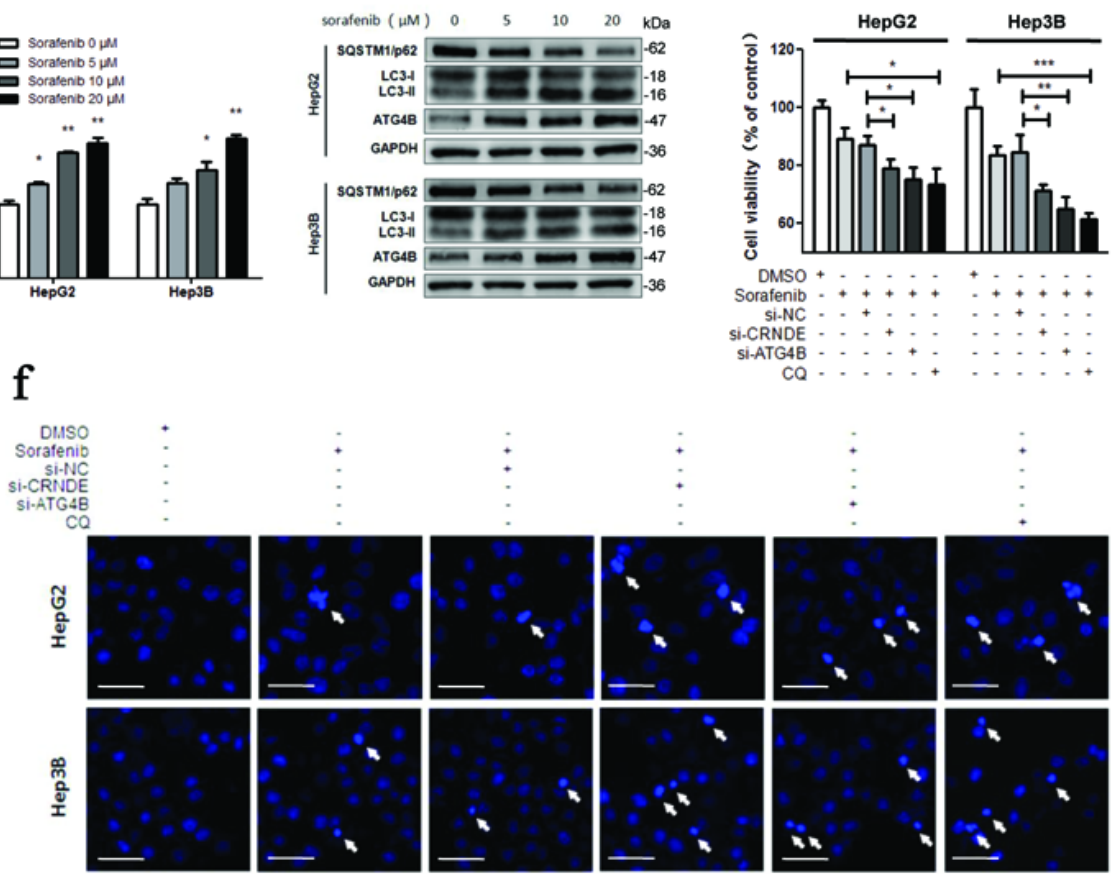

g

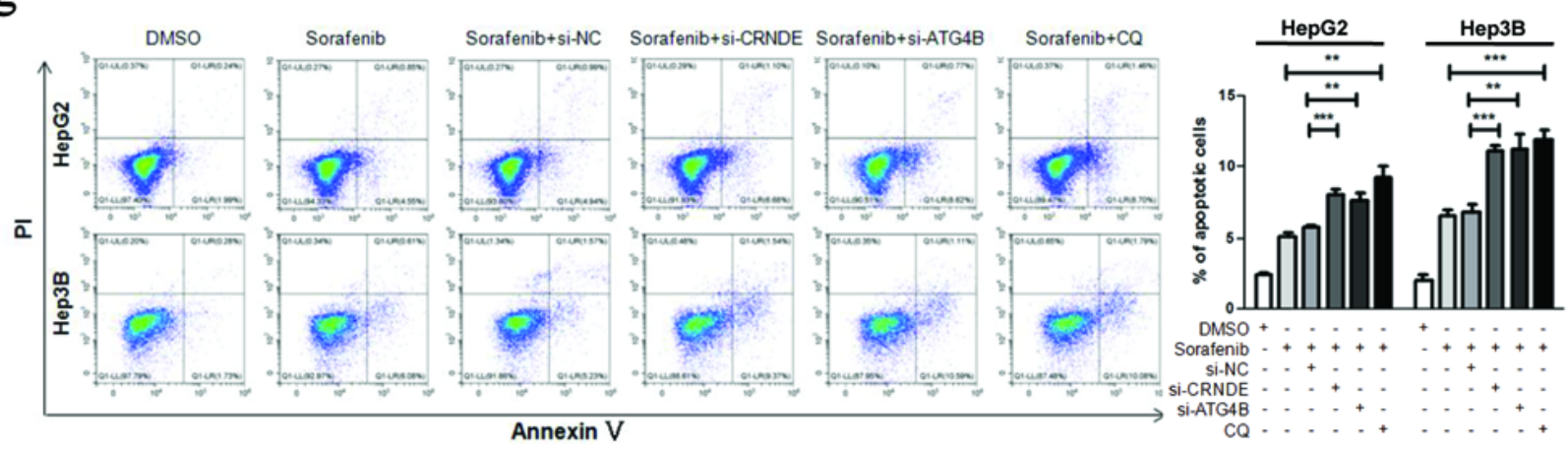

h

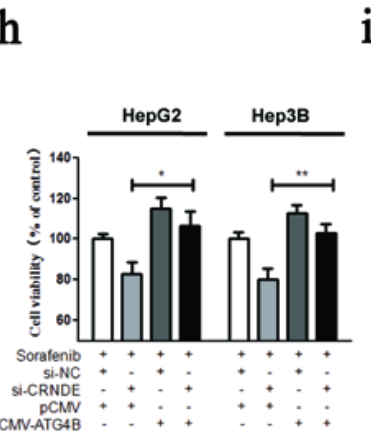

i

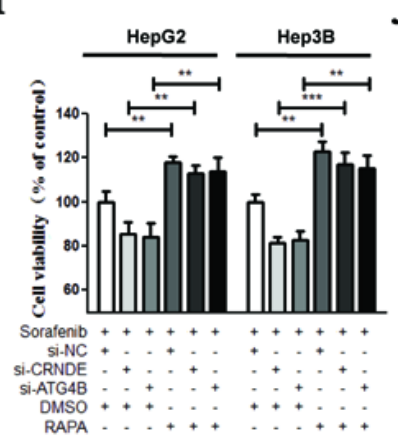

$\mathbf{j}$

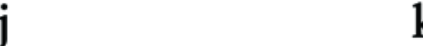

k

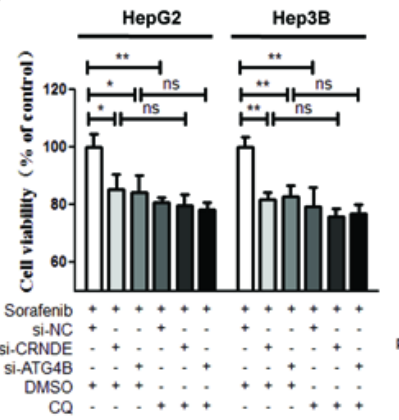

\section{Figure 6}

The CRNDE/ATG4B/autophagy axis attenuates the sensitivity of sorafenib in HCC cells. a-c HepG2 and Hep3B cells were treated with various concentrations of sorafenib for $24 \mathrm{~h}$. Then the levels of CRNDE and ATG4B mRNA were determined by qPCR, and the protein levels of SQSTM1/p62, LC3 and ATG4B were analyzed by Western blot. d HepG2 and Hep3B cells were transfected with si-CRNDE, si-ATG4B or si-NC, or pretreated with $20 \mu \mathrm{M}$ CQ or vehicle control (0.1\% DMSO) for $2 \mathrm{~h} .12 \mathrm{~h}$ later, the cells were treated with 
sorafenib $(10 \mu \mathrm{M})$ for $24 \mathrm{~h}$ and the cell viability was measured by CCK-8 assay. e After treatment as in d, the levels of cleaved PARP, SQSTM1/p62, LC3 and ATG4B were tested by Western blot. $f$ After treatment as in d, HepG2 and Hep3B cells were stained with Hoechst 33258 and observed under a fluorescence microscope (Scale bar: $20 \mu \mathrm{m}$ ). g After treatment as in d, the cells were stained with annexin V-FITC/PI and apoptosis was assayed using flow cytometry. $\mathrm{h} 12 \mathrm{~h}$ after co-transfection with si-CRNDE (or si-NC) and pCMV-ATG4B (or pCMV), HepG2 and Hep3B cells were treated with sorafenib (10 $\mu \mathrm{M})$ for $24 \mathrm{~h}$. Then the cell viability was detected by CCK-8 assay. i $12 \mathrm{~h}$ after transfection with si-CRNDE, si-ATG4B or si-NC), HepG2 and Hep3B cells were co-treated with $0.1 \mu \mathrm{M}$ RAPA (or 0.1\% DMSO) and sorafenib (10 $\mu \mathrm{M})$ for 24 h. Subsequently, the cell viability was detected by CCK-8 assay. j HepG2 and Hep3B cells were transfected with si-CRNDE, si-ATG4B or si-NC. $12 \mathrm{~h}$ after transfection (for CQ co-treatment groups, $10 \mathrm{~h}$ after transfection, the cells were pre-treated with $20 \mu \mathrm{M}$ CQ or $0.1 \%$ DMSO for $2 \mathrm{~h}$ ), the cells were treated with sorafenib $(10 \mu \mathrm{M})$ for $24 \mathrm{~h}$ and the cell viability was determined by CCK-8 assay. k HepG2 and Hep3B cells were transfected with pcDNA-CRNDE or pCMV-ATG4B. $12 \mathrm{~h}$ after transfection (for CQ co-treatment groups, $10 \mathrm{~h}$ after transfection, the cells were pre-treated with $20 \mu \mathrm{M}$ CQ or $0.1 \%$ DMSO for $2 \mathrm{~h}$ ), the cells were treated with sorafenib $(10 \mu \mathrm{M})$ for $24 \mathrm{~h}$ and the cell viability was tested by CCK-8 assay. CQ: chloroquine, RAPA: rapamycin, ns: no significance; $* P<0.05 ; * \star P<0.01 ; * \star * P<0.001$. 
a

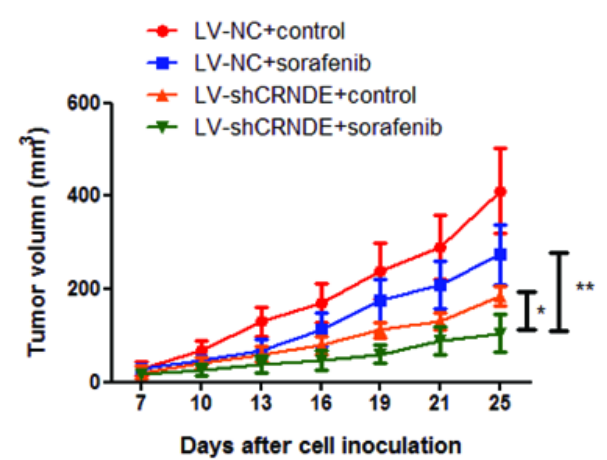

c

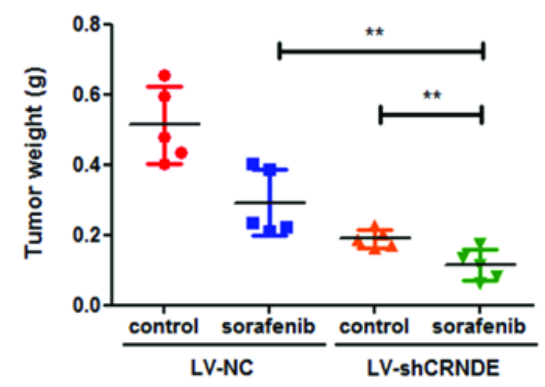

e

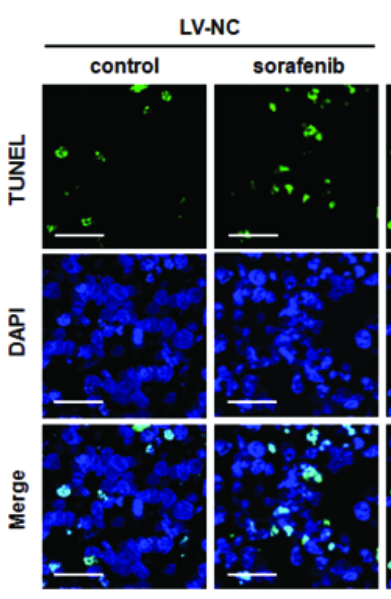

f

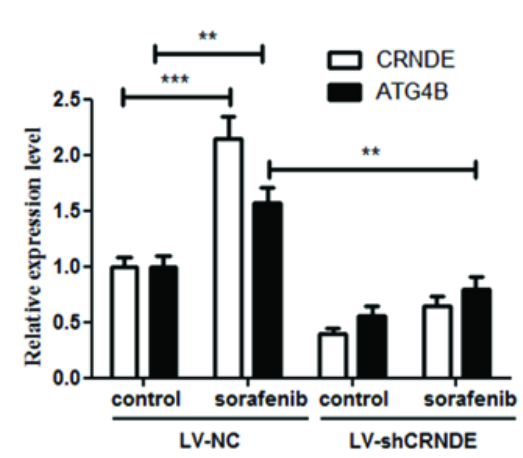

g b

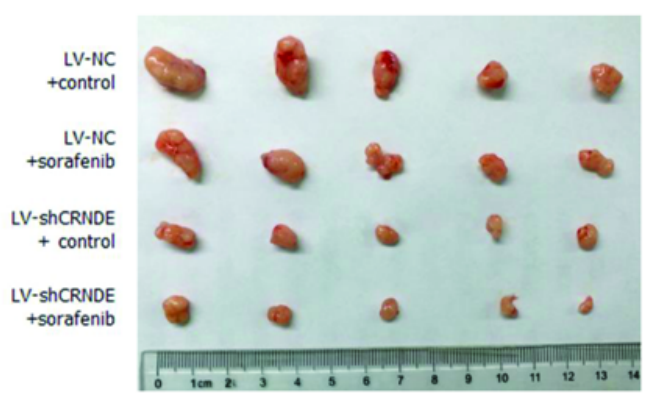

d
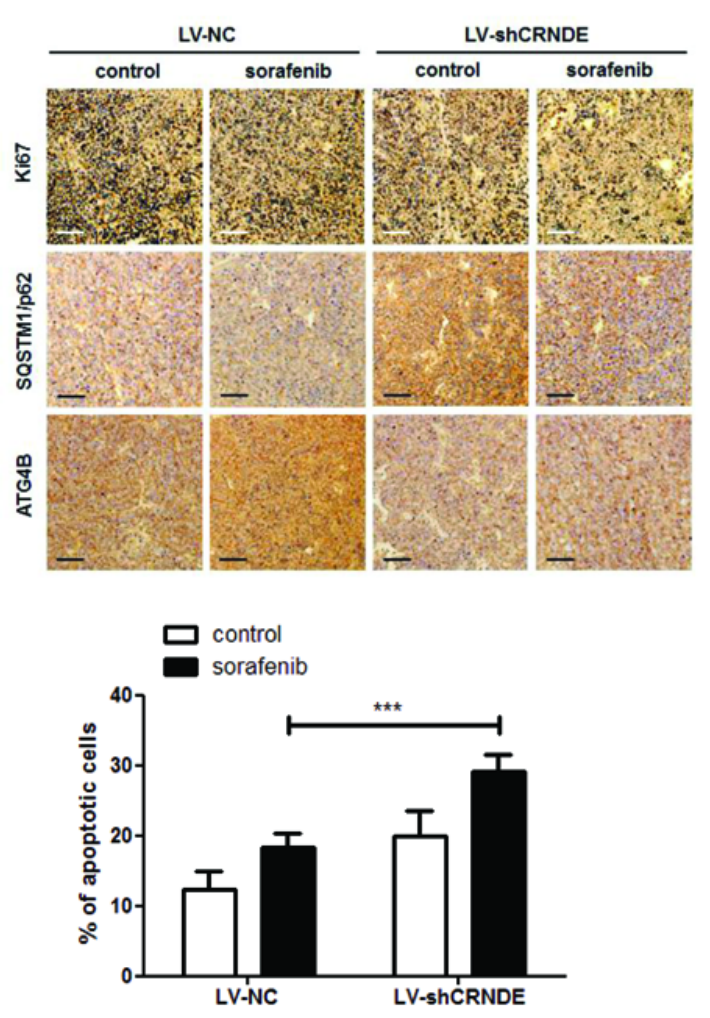

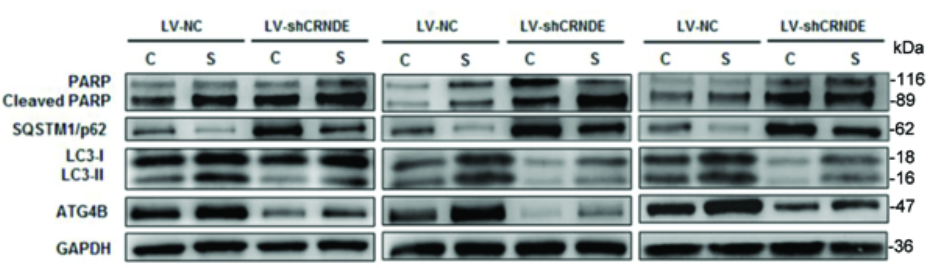

Figure 7

Knockdown of CRNDE sensitizes HCC cells to sorafenib in vivo. The HepG2 cells ( $5 \times 106$ cells in $0.1 \mathrm{~mL}$ PBS for each mouse) with or without stable knockdown of CRNDE (LV-shCRNDE or LV-NC) were subcutaneously injected into the right flank of each nude mouse ( $n=10$ per group). One week after inoculation, the mice in each group were randomized into two subgroups ( $n=5$ per subgroup) and given daily administration of sorafenib $(30 \mathrm{mg} / \mathrm{kg})$ or vehicle control by gavage. The size of xenograft tumors 
was measured every three days and the volume was calculated with the following formula: volume $=$ width $2 \times$ length $\times 1 / 2$ (a). Twenty-five days after inoculation, the xenograft tumors were excised for photographing (b) and weighting (c). The levels of Ki67, SQSTM1/p62 and ATG4B were detected by immunohistochemical staining (d) and apoptosis assay was performed using TUNEL staining (Scale bar: $20 \mu \mathrm{m})(\mathrm{e})$. The levels of CRNDE and ATG4B mRNA were examined by qPCR (f), and the levels of cleaved PARP, SQSTM1/p62, LC3 and ATG4B protein were determined by Western blot (g) using 3 xenograft samples of each group. C: control, S: sorafenib. ${ }^{* * P<0.01 ; ~}{ }^{* \star *} P<0.001$.

\section{Supplementary Files}

This is a list of supplementary files associated with this preprint. Click to download.

- SupplementaryfigureS1.tif

- SupplementaryfigureS2.tif

- SupplementaryfigureS3.tif

- SupplementaryfigureS4.tif

- SupplementaryfigureS5.tif

- SupplementaryfigureS6.tif

- SupplementaryfigureS7.tif

- SupplementaryMaterials.docx 